\title{
Relations on $\bar{M}_{g, n}$ via equivariant Gromov-Witten theory of $\mathbb{P}^{1}$
}

\author{
Felix Janda
}

\begin{abstract}
We give a proof of Pixton's generalized Faber-Zagier relations in the tautological Chow ring of $\bar{M}_{g, n}$. The strategy is very similar to the work of Pandharipande-PixtonZvonkine, who have given a proof of the same result in cohomology. The main tool is the Givental-Teleman classification of semisimple cohomological field theories. While in general only known in cohomology, the Givental-Teleman classification is also valid in the Chow ring for the equivariant Gromov-Witten theory of the projective line, as can be shown via virtual localization. We obtain the relations just from the latter theory.
\end{abstract}

\section{Introduction}

The study of the Chow ring of the moduli space $M_{g}$ of genus $g$ algebraic curves was initiated by Mumford in [Mum83]. For this, he introduced tautological classes, which reflect the geometry of the objects parametrized by the moduli space. The tautological $\operatorname{ring} R^{*}\left(M_{g}\right)$ is the ring generated by tautological classes, and the definitions were later extended to the Deligne-Mumford compactification $\bar{M}_{g, n}$ of stable curves of genus $g$ with $n$ markings, where $R^{*}\left(\bar{M}_{g, n}\right)$ is compactly defined [FP05] as the smallest system

$$
R^{*}\left(\bar{M}_{g, n}\right) \subseteq A^{*}\left(\bar{M}_{g, n}\right)
$$

of subrings compatible with push-forward under the tautological maps, that is, the maps obtained from forgetting marked points or gluing curves along markings. See the recent survey article [Pan16] for a discussion of the tautological ring and the topics surrounding this paper.

There is an explicit set of generators of the ring $R^{*}\left(\bar{M}_{g, n}\right)$ of the form

$$
\xi_{\Gamma *}\left(\prod_{v \in \Gamma} P_{v}\right),
$$

which we briefly describe now. Let $\Gamma$ be a dual graph describing a stratum of $\bar{M}_{g, n}$ by the topological type of its generic elements; that is, the vertices $v$ of $\Gamma$ correspond to irreducible components and are labeled by a genus $g(v)$, edges correspond to nodes, and there are numbered legs corresponding to the markings. Each edge is thought of as glued from two half-edges and

Received 18 October 2015, accepted in final form 5 August 2016.

2010 Mathematics Subject Classification 14H10, 14 N35.

Keywords: moduli of curves, tautological ring, cohomological field theories.

This journal is (c) Foundation Compositio Mathematica 2017. This article is distributed with Open Access under the terms of the Creative Commons Attribution Non-Commercial License, which permits non-commercial reuse, distribution, and reproduction in any medium, provided that the original work is properly cited. For commercial re-use, please contact the Foundation Compositio Mathematica.

The author was supported by the Swiss National Science Foundation grant SNF 200021_143274. 
the legs also count as half-edges. Corresponding to $\Gamma$, there is a gluing map

$$
\xi_{\Gamma}: \prod_{v \in \Gamma} \bar{M}_{g(v), n(v)} \rightarrow \bar{M}_{g, n}
$$

finite of degree $|\operatorname{Aut}(\Gamma)|$, where $n(v)$ is the number of half-edges at vertex $v$. The $P_{v}$ in (1.1) are arbitrarily chosen monomials in the first Chern classes $\psi_{1}, \ldots, \psi_{n(v)}$ of the cotangent line bundles at the markings and the $\kappa$-classes

$$
\kappa_{i}=\pi_{*}\left(\psi_{n(v)+1}^{i+1}\right)
$$

where $\pi$ is the forgetful map

$$
\pi: \bar{M}_{g(v), n(v)+1} \rightarrow \bar{M}_{g(v), n(v)}
$$

See [GP03, Appendix A] for a review of these generators of the tautological ring. There, it is shown how multiplication, push-forward and pull-back under the tautological maps can be defined on the level of generators. In [Pix12], the strata algebra $\mathcal{S}_{g, n}$ is defined as the formal algebra generated by formal classes of the form (1.1). The discussion of [GP03, Appendix A] defines the ring structure on $\mathcal{S}_{g, n}$ and lifts of the tautological maps under the natural forgetful homomorphism $q: \mathcal{S}_{g, n} \rightarrow R^{*}\left(\bar{M}_{g, n}\right)$.

While there is an explicit set of generators, the set of relations between the generators is not known despite much study. The conjectures of Faber [Fab99, FP00] would give a description of the relations. However, recently, Petersen and Tommasi [PT14] gave counter-examples to the Gorenstein conjecture, which is the only one of Faber's conjectures that had remained unproven. On the other hand, Pixton's set [Pix12] of generalized Faber-Zagier relations gives a well-tested conjectural description for the set of tautological relations.

We now give a brief description of Pixton's relations. As for the original relations of Faber and Zagier in $R^{*}\left(M_{g}\right)$, the main input to the formulation of the relations of Pixton are the hypergeometric series

$$
\begin{aligned}
& A(z)=\sum_{i=0}^{\infty} \frac{(6 i) !}{(3 i) !(2 i) !} \frac{(-z)^{i}}{1728^{i}}=1-\frac{5}{144} z \pm \cdots \\
& B(z)=\sum_{i=0}^{\infty} \frac{(6 i) !}{(3 i) !(2 i) !} \frac{1+6 i}{1-6 i} \frac{(-z)^{i}}{1728^{i}}=1+\frac{7}{144} z \mp \cdots .
\end{aligned}
$$

The functions $A$ and $B$ have appeared in many different ways in the study of the moduli space of curves and are strongly related to the asymptotic expansion of the Airy function. See [BJP15] for a review of some of these occurrences.

Given a collection $\left(a_{1}, \ldots, a_{n}\right) \in\{0,1\}^{n}$, Pixton constructs from these series certain elements

$$
\sum_{\Gamma} \frac{1}{|\operatorname{Aut}(\Gamma)|} \frac{1}{2^{h_{1}(\Gamma)}} \xi_{\Gamma *}\left(\left[\prod_{v} \kappa_{v} \prod_{i} B_{i} \prod_{e} \Delta_{e}\right]_{\prod_{v} \zeta_{v}^{g(v)-1}}\right)
$$

in the strata algebra $\mathcal{S}_{g, n}$, where the sum is over all dual graphs $\Gamma$ and the products are over all vertices $v$, markings $i$ and edges $e$ of $\Gamma$. At each vertex $v$, a parity condition is singled out using a variable $\zeta_{v}$ satisfying $\zeta_{v}^{2}=1$. The objects $\kappa_{v}, B_{i}$ and $\Delta_{e}$ in (1.3) depend on the $\zeta$-variables and the square brackets single out a specific coefficient in these variables. Define

$$
B_{i}= \begin{cases}A\left(\zeta_{i} \psi_{i}\right) & \text { if } a_{i}=0 \\ B\left(\zeta_{i} \psi_{i}\right) & \text { if } a_{i}=1\end{cases}
$$




\section{Relations on $\bar{M}_{g, n}$ Via equivariant Gromov-Witten theory of $\mathbb{P}^{1}$}

where $\zeta_{i}$ is $\zeta_{v}$ if the marking $i$ is at vertex $v$, and define

$$
\Delta_{e}=\frac{\zeta^{\prime}+\zeta^{\prime \prime}-A\left(\zeta^{\prime} \psi^{\prime}\right) \zeta^{\prime \prime} B\left(\zeta^{\prime \prime} \psi^{\prime \prime}\right)-\zeta^{\prime} B\left(\zeta^{\prime} \psi^{\prime}\right) A\left(\zeta^{\prime \prime} \psi^{\prime \prime}\right)}{\psi^{\prime}+\psi^{\prime \prime}},
$$

where $\psi^{\prime}$ and $\psi^{\prime \prime}$ are the cotangent line classes at both sides of the node corresponding to $e$, and $\zeta^{\prime}$ and $\zeta^{\prime \prime}$ are the parity variables at the vertices connected by $e$. Finally, $\kappa_{v}$ is the polynomial in formal $\kappa$-classes of $\bar{M}_{g(v), n(v)}$ defined by

$$
\kappa_{v}=\sum_{k=0}^{\infty} \pi_{*}\left(\prod_{i=n(v)+1}^{n(v)+k}\left(\psi_{i}-\psi_{i} A\left(\zeta_{v} \psi_{i}\right)\right)\right),
$$

where $\pi$ forgets the last $k$ markings, via Faber's formula [AC96].

In this paper, we give a proof of the following result, which has been conjectured by Pixton in $[\operatorname{Pix} 12]$.

TheOREm 1.1. Let $(g, n)$ be in the stable range $2 g-2+n>0$, and let $d \geqslant 0$ satisfy the condition

$$
3 d>g-1+\sum_{i=1}^{n} a_{i} .
$$

Then the degree $d$ part of (1.3) is a tautological relation, that is, lies in the kernel of the forgetful $\operatorname{map} q: \mathcal{S}_{g, n} \rightarrow R^{*}\left(\bar{M}_{g, n}\right)$.

Pixton furthermore conjectures that the smallest ideal in $\mathcal{S}_{g, n}$ including the relations of Theorem 1.1 and stable under push-forward under the tautological maps coincides with the set of all tautological relations.

In [PPZ15], Theorem 1.1 is proven on the level of cohomology; in other words, it is proven that (1.3) is mapped to zero under the composition of $q$ and the cycle class map. The main ingredient of the proof of [PPZ15] is the Givental-Teleman classification of semisimple cohomological field theories (CohFTs) applied in the example of Witten's 3-spin class. All arguments of [PPZ15] also apply to Chow rings except for the fact that the classification cannot be used since its proof by Teleman uses topological arguments in an essential way.

For the proof of Theorem 1.1, we will use the same arguments as in [PPZ15] except that we use another CohFT, namely the equivariant Gromov-Witten theory of the projective line. In this example, the classification is well known to be valid also in the Chow setting by a localization calculation [Giv01b] of Givental. The CohFT is slightly more complicated than the CohFT of Witten's 3-spin class in that it depends on one additional parameter. We will, however, see that under a particular specialization of the parameters, it agrees with the CohFT of Witten's 3-spin class.

The CohFT that we use can also alternatively be defined using the moduli space of stable quotients to $\mathbb{P}^{1}$ [MOP11]. Virtual localization for this moduli space has been used in [PP13] to give the first proof of the Faber-Zagier relations of $R^{*}\left(M_{g}\right)$. The current work can be viewed as a simplification of the author's first proof [Jan13] of Theorem 1.1 generalizing the arguments of [PP13]. We prefer to use Gromov-Witten theory over stable quotients here because the reconstruction of the CohFT in the Chow ring via localization had already essentially been proven by Givental [Giv01b].

Instead of $\mathbb{P}^{1}$, we could also have studied the Gromov-Witten theory of higher-dimensional projective spaces in order to find relations in the tautological ring. The resulting relations become 
increasingly complicated with rising dimension. Still, in [Jan15] it is shown that all of them can be expressed in terms of Pixton's relations.

\section{Plan of the paper}

We start by reviewing standard material on CohFTs including the Givental-Teleman reconstruction in Section 2. In Section 3, we restrict ourselves to the example of the equivariant GromovWitten theory of $\mathbb{P}^{1}$, studying first the Frobenius algebra underlying the theory in Section 3.2 and the $R$-matrix in Section 3.3. In Section 3.4, we observe that under a particular specialization of parameters, this data coincides with the 3-spin theory. We use this in Section 3.5 to conclude Theorem 1.1.

In the appendix, we make explicit Givental's proof of the reconstruction of the GromovWitten theory of projective spaces and we recall his mirror symmetry description of the small theories.

\section{Cohomological field theories}

\subsection{Definitions}

Cohomological field theories were first introduced by Kontsevich and Manin in [KM97] to formalize the structure of classes from Gromov-Witten theory. Let $V$ be an $N$-dimensional $\mathbb{C}$-vector space and $\eta$ a nonsingular bilinear form on $V$.

Definition 2.1. A cohomological field theory (CohFT) $\Omega$ on $(V, \eta)$ (on the level of the Chow ring) is a system

$$
\Omega_{g, n} \in A^{*}\left(\bar{M}_{g, n}\right) \otimes\left(V^{*}\right)^{\otimes n}
$$

of multilinear forms with values in the Chow ring of $\bar{M}_{g, n}$ satisfying the following properties:

(i) ( $S_{n}$-equivariance) The multilinear form $\Omega_{g, n}$ is invariant with respect to the $S_{n}$-action permuting the factors of $\left(V^{*}\right)^{\otimes n}$ and the marked points simultaneously.

(ii) (Gluing) The pull-back of $\Omega_{g, n}$ via the gluing map

$$
\bar{M}_{g_{1}, n_{1}+1} \times \bar{M}_{g_{2}, n_{2}+1} \rightarrow \bar{M}_{g, n}
$$

is given by the direct product of $\Omega_{g_{1}, n_{2}+1}$ and $\Omega_{g_{2}, n_{2}+1}$ with the bivector $\eta^{-1}$ inserted at the two points glued together. Similarly, for the gluing map $\bar{M}_{g-1, n+2} \rightarrow \bar{M}_{g, n}$, the pull-back of $\Omega_{g, n}$ is given by $\Omega_{g-1, n+2}$ with $\eta^{-1}$ inserted at the two points glued together.

(iii) (Unit) There is a special element $\mathbf{1} \in V$ called the unit such that

$$
\Omega_{g, n+1}\left(v_{1}, \ldots, v_{n}, \mathbf{1}\right)
$$

is the pull-back of $\Omega_{g, n}\left(v_{1}, \ldots, v_{n}\right)$ under the forgetful map and

$$
\Omega_{0,3}(v, w, \mathbf{1})=\eta(v, w) .
$$

DeFinition 2.2. The quantum product $(u, v) \mapsto u v$ on $V$ with unit $\mathbf{1}$ is defined by the condition

$$
\eta(u v, w)=\Omega_{0,3}(u, v, w) .
$$

Definition 2.3. A CohFT is called semisimple if the algebra $V$ is semisimple, that is, if it admits a basis of orthogonal idempotent elements. 


\section{Relations on $\bar{M}_{g, n}$ Via equivariant Gromov-Witten theory of $\mathbb{P}^{1}$}

Remark 2.4. Many CohFTs come in families. The definition of a CohFT directly generalizes to the setting where $V$ is a module over a commutative ring $T$ by demanding $T$-linearity instead of $\mathbb{C}$-linearity.

\subsection{Examples}

EXAMPLE 2.5. For each Frobenius algebra, there is the trivial CohFT (also called topological field theory or TQFT) $\Omega_{g, n}$ characterized by (2.1) and the condition

$$
\Omega_{g, n} \in A^{0}\left(\bar{M}_{g, n}\right) \otimes\left(V^{*}\right)^{\otimes n} .
$$

Let us record an explicit formula for the appendix: In the case that the Frobenius algebra is semisimple, there is a basis $\epsilon_{i}$ of orthogonal idempotents of $V$ and

$$
\tilde{\epsilon}_{i}=\frac{\epsilon_{i}}{\sqrt{\Delta_{i}}}
$$

where $\Delta_{i}=\eta\left(\epsilon_{i}, \epsilon_{i}\right)$, is a corresponding orthonormal basis of normalized idempotents. It is not difficult to show

$$
\Omega_{g, n}\left(\tilde{\epsilon}_{i_{1}}, \ldots, \tilde{\epsilon}_{i_{n}}\right)= \begin{cases}\sum_{j} \Delta_{i_{j}}^{g-1} & \text { if } n=0, \\ \Delta_{i_{1}}^{\frac{2 g-2+n}{2}} & \text { if } i_{1}=\cdots=i_{n}, \\ 0 & \text { else, }\end{cases}
$$

since, in general, $\Omega_{0,3}$ is uniquely determined by $\eta$ and the quantum product, and the other $\Omega_{g, n}$ are determined by considering the restriction to the locus of curves glued together from $2 g-2+n$ rational curves with three special points.

Example 2.6. The Chern polynomial $c_{t}(\mathbb{E})$ of the Hodge bundle $\mathbb{E}$ gives a one-dimensional CohFT over the ring $\mathbb{Q}[t]$.

EXAMPLE 2.7. Let $X$ be a smooth, projective variety such that the cycle class map gives an isomorphism between Chow and cohomology rings. Then the Gromov-Witten theory of $X$ defines a CohFT based on the module $A^{*}(X) \otimes N$ over the Novikov ring $N:=\mathbb{C} \llbracket q^{\beta} \rrbracket$, which is generated by formal variables $q^{\beta}$ indexed by effective, integral curve classes $\beta$. The CohFT is defined by setting, for $v_{1}, \ldots, v_{n} \in A^{*}(X)$,

$$
\Omega_{g, n}\left(v_{1}, \ldots, v_{n}\right)=\sum_{\beta} p_{*}\left(\prod_{i=1}^{n} \operatorname{ev}_{i}^{*}\left(v_{i}\right) \cap\left[\bar{M}_{g, n}(X, \beta)\right]^{\mathrm{vir}}\right) q^{\beta},
$$

where the sum ranges over effective, integral curve classes, $\mathrm{ev}_{i}$ is the $i$ th evaluation map and $p$ is the forgetful map $p: \bar{M}_{g, n}(X, \beta) \rightarrow \bar{M}_{g, n}$. The gluing property follows from the splitting axiom of virtual fundamental classes. The fundamental class of $X$ is the unit of the CohFT, and the unit axioms follow from the identity axiom in Gromov-Witten theory.

For a torus action on $X$, this example can be enhanced to give a CohFT from the $T$-equivariant Gromov-Witten theory of $X$. In this case, the CohFT is defined on the module $A_{T}^{*}(X) \otimes N$. The case that $X=\mathbb{P}^{1}$ with a $\mathbb{C}^{*}$-action will be the main example we will study. We will make the definition of the CohFT more concrete in Section 3.1.

\subsection{Reconstruction}

The (upper half of the) symplectic loop group corresponding to a vector space $V$ with nonsingular bilinear form $\eta$ is the group of endomorphism-valued power series $V \llbracket z \rrbracket$ such that the symplectic 
condition

$$
R(z) R^{t}(-z)=1
$$

holds. Here $R^{t}$ is the adjoint of $R$ with respect to $\eta$. There is an action of this group on the space of all CohFTs based on a fixed semisimple Frobenius algebra structure of $V$. The action is often named after Givental because he introduced it on the level of arbitrary genus Gromov-Witten potentials.

Given a CohFT $\Omega_{g, n}$ and such an endomorphism $R$, the new CohFT $R \Omega_{g, n}$ takes the form of a sum over dual graphs $\Gamma$

$$
R \Omega_{g, n}\left(v_{1}, \ldots, v_{n}\right)=\sum_{\Gamma} \frac{1}{\operatorname{Aut}(\Gamma)} \xi_{*}\left(\prod_{v} \sum_{k=0}^{\infty} \frac{1}{k !} \pi_{*} \Omega_{g(v), n(v)+k}(\ldots)\right),
$$

where $\xi: \prod_{v} \bar{M}_{g(v), n(v)} \rightarrow \bar{M}_{g, n}$ is the gluing map of curves of topological type $\Gamma$ from their irreducible components, $\pi: \bar{M}_{g(v), n(v)+k} \rightarrow \bar{M}_{g(v), n(v)}$ forgets the last $k$ markings and we still need to specify the arguments of $\prod_{v} \Omega_{g(v), n(v)+k}$. Instead of allowing only vectors in $V$ to be put into $\Omega_{g, n}$, we will allow elements of $V \llbracket \psi_{1}, \ldots, \psi_{n} \rrbracket$, where $\psi_{i}$ acts on the cohomology of the relevant moduli space of curves by multiplication with the $i$ th cotangent line class. The arguments of $\prod_{v} \Omega_{g(v), n(v)+k}$ are prescribed by the following:

- Into each argument corresponding to a marking of the curve, put $R^{-1}(\psi)$ applied to the corresponding vector.

- Into each pair of arguments corresponding to an edge, put the bivector

$$
\frac{R^{-1}\left(\psi_{1}\right) \otimes R^{-1}\left(\psi_{2}\right)-\operatorname{Id} \otimes \operatorname{Id}}{-\psi_{1}-\psi_{2}} \eta^{-1} \in V^{\otimes 2} \llbracket \psi_{1}, \psi_{2} \rrbracket,
$$

where one has to substitute the $\psi$-classes at each side of the normalization of the node for $\psi_{1}$ and $\psi_{2}$. By the symplectic condition this is well defined.

- At each of the additional arguments for each vertex, put

$$
T(\psi):=\psi\left(\operatorname{Id}-R^{-1}(\psi)\right) \mathbf{1},
$$

where $\psi$ is the cotangent line class corresponding to that vertex. Since $T(z)=O\left(z^{2}\right)$, the above $k$-sum is finite.

The following reconstruction result (on the level of potentials) was first proposed by Givental [Giv01a].

TheOREm 2.8 ([Tel12]). In cohomology, the $R$-matrix action is free and transitive on the space of semisimple CohFTs based on a given Frobenius algebra. In particular, for any CohFT $\Omega$ with underlying TQFT $\omega$, there exists an $R$-matrix $R$ such that $\Omega=R \omega$.

Remark 2.9. Teleman's proof relies heavily on topological results (Mumford's conjecture/Madsen-Weiss theorem) and it is therefore not known if the same classification result also holds in general when we work with Chow rings instead of cohomology.

Remark 2.10. In the appendix, we review a virtual localization computation of Givental which implies that Theorem 2.8 is true in the Chow ring for the equivariant Gromov-Witten theory of projective spaces. Furthermore, mirror symmetry gives an explicit description of the $R$-matrix.

ExAmple 2.11. By Mumford's Grothendieck-Riemann-Roch calculation [Mum83], the single entry of the $R$-matrix taking the trivial one-dimensional CohFT to the CohFT from Example 2.6 


\section{Relations on $\bar{M}_{g, n}$ Via Equivariant Gromov-Witten theory of $\mathbb{P}^{1}$}

is given by

$$
\exp \left(\sum_{i=1}^{\infty} \frac{B_{2 i}}{2 i(2 i-1)}(t z)^{2 i-1}\right)
$$

where the $B_{2 i}$ are the Bernoulli numbers, defined by

$$
\sum_{i=0}^{\infty} B_{i} \frac{x^{i}}{i !}=\frac{x}{e^{x}-1}
$$

More generally, if we consider a more general CohFT given by a product of Chern polynomials (in different variables) of the Hodge bundle, the $R$-matrix from the trivial CohFT is the product of the $R$-matrices of the factors.

\section{Equivariant Gromov-Witten theory of $\mathbb{P}^{1}$}

In this section, we consider the projective line $\mathbb{P}^{1}$ together with a $\mathbb{C}^{*}$-action with weights $(0,1)$ and the tautological relations resulting from it.

\subsection{Definition of the CohFT}

We make the CohFT described in Example 2.7 more concrete in our example.

The only effective curve classes on $\mathbb{P}^{1}$ are nonnegative multiples of $\left[\mathbb{P}^{1}\right]$. Hence the Novikov ring is the ring $\mathbb{C} \llbracket q \rrbracket$, where $q$ corresponds to $\left[\mathbb{P}^{1}\right]$.

Let $\lambda$ be the first Chern class of the dual of the one-dimensional vector space with a $\mathbb{C}^{*}$-action of weight 1 . Then the equivariant Chow ring of $\mathbb{P}^{1}$ is isomorphic to

$$
A_{\mathbb{C}^{*}}^{*}\left(\mathbb{P}^{1}\right) \cong \mathbb{Q}[\lambda, H] / H(H-\lambda) ;
$$

this expresses the fact that the equivariant classes $H$ and $H-\lambda$ of the two fixed points 0 and $\infty$ intersect trivially. The module $A_{\mathbb{C}^{*}}^{*}\left(\mathbb{P}^{1}\right) \otimes N$ over $\mathbb{C}[\lambda] \otimes N$ is the state space of the CohFT.

The CohFT $\Omega_{g, n}^{\mathbb{P}^{1}}$ corresponding to the equivariant Gromov-Witten theory of $\mathbb{P}^{1}$ is defined by setting, for $v_{1}, \ldots, v_{n} \in A_{\mathbb{C}^{*}}^{*}\left(\mathbb{P}^{1}\right)$,

$$
\Omega_{g, n}\left(v_{1}, \ldots, v_{n}\right)=\sum_{d=0}^{\infty} p_{*}\left(\prod_{i=1}^{n} \operatorname{ev}_{i}^{*}\left(v_{i}\right) \cap\left[\bar{M}_{g, n}\left(\mathbb{P}^{1}, d\right)\right]^{\mathrm{vir}}\right) q^{d},
$$

where $\bar{M}_{g, n}\left(\mathbb{P}^{1}, d\right)$ is the moduli space of stable maps of degree $d$ to $\mathbb{P}^{1}$ and $p$ is the forgetful map to $\bar{M}_{g, n}$. The dimension of the virtual class $\left[\bar{M}_{g, n}\left(\mathbb{P}^{1}, d\right)\right]^{\text {vir }}$ is

$$
\left(3-\operatorname{dim} \mathbb{P}^{1}\right)(g-1)+n+\left\langle d H, c_{1}\left(T_{\mathbb{P}^{1}}\right)\right\rangle=2 g-2+n+2 d .
$$

This means that for any $a_{1}, \ldots, a_{n} \in\{0,1\}$, the coefficient of $q^{d}$ in $\Omega_{g, n}\left(H^{a_{1}}, \ldots, H^{a_{n}}\right)$ has degree

$$
3 g-3+n-(2 g-2+n+2 d)+\sum_{i=1}^{n} a_{i}=g-1-2 d+\sum_{i=1}^{n} a_{i} .
$$

Since this integer is negative for large enough $d$, the sum over $d$ in (3.1) is finite and defines a CohFT on the module $A_{\mathbb{C}^{*}}^{*}\left(\mathbb{P}^{1}\right)[q]$ over the ring $\mathbb{C}[\lambda, q]$. 


\section{F. JANDA}

\subsection{The underlying TQFT}

The nonsingular bilinear form on $A_{T}^{*}\left(\mathbb{P}^{1}\right)$ is given by the equivariant Poincaré pairing. In the basis $\{1, H\}$, it takes the form

$$
\left(\begin{array}{ll}
0 & 1 \\
1 & \lambda
\end{array}\right)
$$

Define

$$
\widetilde{H}:=H-\frac{\lambda}{2},
$$

so that in the basis $\{1, \widetilde{H}\}$, the Poincaré pairing becomes just

$$
\left(\begin{array}{ll}
0 & 1 \\
1 & 0
\end{array}\right)
$$

The equivariant quantum product of $\mathbb{P}^{1}$ is defined using three-pointed genus zero equivariant Gromov-Witten invariants as in (2.1). Explicitly, the relation defining the classical equivariant cup product gets deformed to

$$
H(H-\lambda)=q
$$

Equivalently, we can write

$$
\widetilde{H}^{2}=\phi, \quad \text { where we set } \phi=\lambda^{2} / 4+q .
$$

Notice that the product is semisimple if and only if $\phi \neq 0$.

By choosing a root $\sqrt{\phi}$ of $\phi$ (working in an extension of the base ring), we can easily write down idempotents

$$
\frac{1}{\Delta_{ \pm}} \widetilde{H}+\frac{1}{2}
$$

where $\Delta_{ \pm}= \pm 2 \sqrt{\phi}$. By choosing further roots $\sqrt{\Delta_{ \pm}}$, we can define normalized idempotents such that the change of basis $\Psi$ from the basis of normalized idempotents to the basis $\{1, \widetilde{H}\}$ is given by

For convenience, we also note that

$$
\Psi=\left(\begin{array}{cc}
\frac{\sqrt{\phi}}{\sqrt{\Delta_{+}}} & \frac{-\sqrt{\phi}}{\sqrt{\Delta_{-}}} \\
\frac{1}{\sqrt{\Delta_{+}}} & \frac{1}{\sqrt{\Delta_{-}}}
\end{array}\right) .
$$

$$
\Psi^{-1}=\left(\begin{array}{cc}
\frac{1}{\sqrt{\Delta_{+}}} & \frac{\sqrt{\phi}}{\sqrt{\Delta_{+}}} \\
\frac{1}{\sqrt{\Delta_{-}}} & \frac{-\sqrt{\phi}}{\sqrt{\Delta_{-}}}
\end{array}\right) .
$$

By induction, we can show that the underlying TQFT $\omega_{g, n}$ of $\Omega_{g, n}^{\mathbb{P}^{1}}$ is explicitly given by

$$
\omega_{g, n}\left(1^{\otimes a}, \widetilde{H}^{\otimes b}\right)= \begin{cases}2^{g} \phi^{\frac{g-1+b}{2}} & \text { if } g-1+b \text { is even }, \\ 0 & \text { else, }\end{cases}
$$

where we have used a shorthand notation for the arguments of $\omega_{g, n}$, meaning $a$ insertions of 1 and $b$ insertions of $\widetilde{H}$. Notice that the TQFT applied to basis vectors takes values in $\mathbb{Q}[\phi]$. 


\section{Relations on $\bar{M}_{g, n}$ Via equivariant Gromov-Witten theory of $\mathbb{P}^{1}$}

\subsection{The $R$-matrix}

As reviewed in the appendix, the $R$-matrix necessary for reconstructing the equivariant GromovWitten theory of $\mathbb{P}^{1}$ can be found by using virtual localization for a nontrivial $\mathbb{C}^{*}$-action and made explicit using stationary phase asymptotics of certain oscillating integrals on the so-called mirror of $\mathbb{P}^{1}$. The coefficients of the $R$-matrix obtained from virtual localization are valued in $\mathbb{Q}\left[\lambda^{ \pm}\right] \llbracket q, z \rrbracket$, but the mirror description shows that the coefficients are obtained by expanding elements of $\mathbb{Q}\left[\lambda^{ \pm}, \phi^{ \pm}\right] \llbracket z \rrbracket$ around $q=0$. In any case, the $R$-matrix is defined over a nontrivial ring extension of $\mathbb{Q}[\lambda, q]$, and it is, at first sight, not clear how this is compatible with the fact that the CohFT is defined over $\mathbb{C}[\lambda, q]$. As we will see in Section 3.5, such a contrast implies the existence of tautological relations.

As reviewed in Section A.6 of the appendix (see also [BJP15]), the $R$-matrix can be computed from the stationary phase asymptotics of the oscillating integrals

$$
\frac{1}{\sqrt{-2 \pi z}} \int_{\Gamma_{ \pm}} e^{F(x) / z}\left(e^{x}-\frac{\lambda}{2}\right)^{1-i} \mathrm{~d} x
$$

where $\Gamma_{ \pm}$are suitable real, one-dimensional cycles and

$$
F(x)=e^{x}+q e^{-x}-\lambda x
$$

is the superpotential. Let $u_{ \pm}$be the critical values at the critical points

$$
e^{x}=\frac{\lambda}{2} \pm \sqrt{\phi}
$$

of $F$. The $\Gamma_{ \pm}$are the corresponding Lefschetz thimbles, each going through exactly one of the critical points.

We start with the stationary phase asymptotics for the integral (3.3) when $i=1$. Shifting each critical point to the origin, rescaling $x$, expanding the exponential and using

$$
\frac{1}{\sqrt{2 \pi}} \int_{-\infty}^{\infty} e^{-\frac{x^{2}}{2}} x^{i} \mathrm{~d} x= \begin{cases}(i-1) ! ! & \text { if } i \text { is even } \\ 0 & \text { else }\end{cases}
$$

gives

$$
\begin{aligned}
& \frac{1}{\sqrt{-2 \pi z}} \int_{\Gamma_{ \pm}} e^{\left(u_{ \pm}+\Delta_{ \pm} \frac{x^{2}}{2}+\lambda \frac{x^{3}}{6}+\Delta_{ \pm} \frac{x^{4}}{24}+\lambda \frac{x^{5}}{120}+\cdots\right) / z} \mathrm{~d} x \\
& \asymp \frac{e^{u_{ \pm}} / z}{\sqrt{\Delta_{ \pm}} \sqrt{2 \pi}} \int_{-\infty}^{\infty} e^{-\frac{x^{2}}{2}} e^{-\frac{x^{3}}{6} \frac{\lambda}{\Delta_{ \pm}^{3 / 2}} \sqrt{-z}-\frac{x^{4}}{24} \frac{1}{\Delta_{ \pm}}(-z)-\frac{x^{5}}{120} \frac{\lambda}{\Delta_{ \pm}^{5 / 2}}(-z)^{3 / 2}-\cdots} \mathrm{d} x \\
& \asymp \frac{e^{u_{ \pm}} / z}{\sqrt{\Delta_{ \pm}} \sqrt{2 \pi}} \int_{-\infty}^{\infty} e^{-\frac{x^{2}}{2}} e^{-\frac{x^{3}}{6}\left(\lambda \sqrt{-z} / \Delta_{ \pm}^{3 / 2}\right)-\frac{x^{4}}{24}(-z) / \Delta_{ \pm}-\frac{x^{5}}{120} \lambda(-z)^{3 / 2} / \Delta_{ \pm}^{5 / 2}-\cdots} \mathrm{d} x \\
& \asymp \frac{e^{u_{ \pm} / z}}{\sqrt{\Delta_{ \pm}}} F_{0}\left(z \frac{\lambda^{2}}{\Delta_{ \pm}^{3}}, \frac{\Delta_{ \pm}^{2}}{\lambda^{2}}\right)
\end{aligned}
$$

for some power series

$$
F_{0}(x, y)=1-\left(\frac{5}{24}-\frac{1}{8} y\right) x+\left(\frac{385}{1152}-\frac{77}{192} y+\frac{9}{128} y^{2}\right) x^{2}+\cdots \in \mathbb{Q}[y] \llbracket x \rrbracket .
$$


When $i=0$, the additional factor

in (3.3) becomes

$$
e^{x}-\frac{\lambda}{2}
$$

$$
\left(\frac{\lambda}{2} \pm \sqrt{\phi}\right) e^{x}-\frac{\lambda}{2}
$$

after translation and

$$
\pm \sqrt{\phi}\left(\left(1+\frac{\lambda}{\Delta_{ \pm}}\right) e^{x \Delta_{ \pm}^{-1 / 2} \sqrt{-z}}-\frac{\lambda}{\Delta_{ \pm}}\right) .
$$

after scaling. So for $i=0$, the asymptotic expansion is given by

$$
\frac{ \pm \sqrt{\phi} e^{u_{ \pm} / z}}{\sqrt{\Delta_{ \pm}}} F_{1}\left(z \frac{\lambda^{2}}{\Delta_{ \pm}^{3}}, \frac{\Delta_{ \pm}^{2}}{\lambda^{2}}\right)
$$

where

$$
F_{1}(x, y)=1+\left(\frac{7}{24}-\frac{3}{8} y\right) x-\left(\frac{455}{1152}-\frac{33}{64} y+\frac{15}{128} y^{2}\right) x^{2}+\cdots \in \mathbb{Q}[y] \llbracket x \rrbracket .
$$

In total, the $S$-matrix from the basis of normalized idempotents to the basis $\{1, \widetilde{H}\}$ is given by

$$
\left(\begin{array}{ll}
\frac{\sqrt{\phi}}{\sqrt{\Delta_{+}}} F_{1}\left(z \frac{\lambda^{2}}{\Delta_{+}^{3}}, \frac{\Delta_{+}^{2}}{\lambda^{2}}\right) & \frac{-\sqrt{\phi}}{\sqrt{\Delta_{-}}} F_{1}\left(z \frac{\lambda^{2}}{\Delta_{-}^{3}}, \frac{\Delta_{-}^{2}}{\lambda^{2}}\right) \\
\frac{1}{\sqrt{\Delta_{+}}} F_{0}\left(z \frac{\lambda^{2}}{\Delta_{+}^{3}}, \frac{\Delta_{+}^{2}}{\lambda^{2}}\right) & \frac{1}{\sqrt{\Delta_{-}}} F_{0}\left(z \frac{\lambda^{2}}{\Delta_{-}^{3}}, \frac{\Delta_{-}^{2}}{\lambda^{2}}\right)
\end{array}\right)\left(\begin{array}{cc}
e^{u_{+}} / z & 0 \\
0 & e^{u_{-} / z}
\end{array}\right) .
$$

Multiplying the first factor from the right by $\Psi^{-1}$ gives the $R$-matrix written in the basis $\{1, \widetilde{H}\}$ :

$$
R(z)=\left(\begin{array}{cc}
F_{1}^{e} & \sqrt{\phi} F_{1}^{o} \\
\frac{1}{\sqrt{\phi}} F_{0}^{o} & F_{0}^{e}
\end{array}\right)\left(z \frac{\lambda^{2}}{\Delta_{+}^{3}}, \frac{\Delta_{+}^{2}}{\lambda^{2}}\right)=\left(\begin{array}{cc}
F_{1}^{e} & \sqrt{\phi} F_{1}^{o} \\
\frac{1}{\sqrt{\phi}} F_{0}^{o} & F_{0}^{e}
\end{array}\right)\left(z \frac{\lambda^{2}}{8 \phi^{3 / 2}}, 4 \frac{\phi}{\lambda^{2}}\right),
$$

where

$$
F_{i}^{e}(x, y)=\frac{F_{i}(x, y)+F_{i}(-x, y)}{2}, \quad F_{i}^{o}(x, y)=\frac{F_{i}(x, y)-F_{i}(-x, y)}{2} .
$$

The symplectic condition (2.2) implies

$$
R^{-1}(z)=\left(\begin{array}{ll}
0 & 1 \\
1 & 0
\end{array}\right) R^{t}(-z)\left(\begin{array}{ll}
0 & 1 \\
1 & 0
\end{array}\right)
$$

So we can easily compute

$$
R^{-1}(z)=\left(\begin{array}{cc}
F_{0}^{e} & \sqrt{\phi} F_{1}^{o} \\
\frac{1}{\sqrt{\phi}} F_{0}^{o} & F_{1}^{e}
\end{array}\right)\left(-z \frac{\lambda^{2}}{8 \phi^{3 / 2}}, 4 \frac{\phi}{\lambda^{2}}\right) .
$$

Remark 3.1. The power series $F_{i}^{e}(x, y)$ are even in $x$; the power series $F_{i}^{o}(x, y)$ are odd. In particular, the coefficients of $R(z)$ in the basis $\{1, \widetilde{H}\}$ are power series in $z$ whose coefficients are Laurent polynomials in $\lambda$ and $\phi$.

\subsection{Limit}

In this section, we show how, out of the CohFT $\Omega_{g, n}^{\mathbb{P}^{1}}$, a CohFT can be extracted that is very similar to the theory of the shifted Witten's 3-spin class as studied in [PPZ15]. 


\section{Relations on $\bar{M}_{g, n}$ Via Equivariant Gromov-Witten theory of $\mathbb{P}^{1}$}

We use the isomorphisms

$$
\mathbb{Q}[\lambda, q] \cong \mathbb{Q}[\lambda, \phi], \quad A_{\mathbb{C}^{*}}^{*}\left(\mathbb{P}^{1}\right) \otimes \mathbb{Q}[q] \cong \mathbb{Q}[\lambda, \phi]\langle 1, \widetilde{H}\rangle
$$

defined by

$$
q \mapsto \phi-\frac{\lambda^{2}}{4}, \quad H \mapsto \widetilde{H}+\frac{\lambda}{2}
$$

once and for all so that the CohFT $\Omega_{g, n}^{\mathbb{P}^{1}}$ is defined over the ring $\mathbb{Q}[\lambda, \phi]$ and based on the module $\mathbb{Q}[\lambda, \phi]\langle 1, \widetilde{H}\rangle$. (It follows from the discussion of Section 3.3 that we can use $\mathbb{Q}$-coefficients (instead of $\mathbb{C}$-coefficients), but it is not of much importance which field we use.)

We can define a new CohFT $\widetilde{\Omega}_{g, n}^{\mathbb{P}^{1}}$ on the module $\mathbb{Q}\left[\lambda^{ \pm}, \phi\right]\langle 1, \widetilde{H}\rangle$ over the ring $\mathbb{Q}\left[\lambda^{ \pm 1}, \phi\right]$ as the composition

$$
\widetilde{\Omega}_{g, n}^{\mathbb{P}^{1}}=\varphi \circ \Omega_{g, n}^{\mathbb{P}^{1}}
$$

where

$$
\varphi: \quad A^{*}\left(\bar{M}_{g, n}\right) \otimes \mathbb{Q}[\lambda, \phi] \rightarrow A^{*}\left(\bar{M}_{g, n}\right) \otimes \mathbb{Q}\left[\lambda^{ \pm}, \phi\right]
$$

is the ring homomorphism induced by

$$
C \mapsto\left(-\frac{4}{3 \lambda^{2}}\right)^{i} C
$$

for any $C \in A^{i}\left(\bar{M}_{g, n}\right)$. By definition, $\varphi$ leaves elements of $A^{0}\left(\bar{M}_{g, n}\right) \otimes \mathbb{Q}[\lambda, \phi]$ invariant and therefore the TQFTs of $\Omega_{g, n}^{\mathbb{P}^{1}}$ and $\widetilde{\Omega}_{g, n}^{\mathbb{P}^{1}}$ are the same (up to the change of base ring). Since the formal variable $z$ of an $R$-matrix measures degree in the Chow ring, we have

$$
\widetilde{R}(z)=R\left(-\frac{4}{3} z \lambda^{-2}\right)=\left(\begin{array}{cc}
F_{1}^{e} & \sqrt{\phi} F_{1}^{o} \\
\frac{1}{\sqrt{\phi}} F_{0}^{o} & F_{0}^{e}
\end{array}\right)\left(-\frac{z}{6 \phi^{3 / 2}}, 4 \frac{\phi}{\lambda^{2}}\right),
$$

where $R(z)$ and $\widetilde{R}(z)$ denote the $R$-matrices of $\Omega_{g, n}^{\mathbb{P}^{1}}$ and $\widetilde{\Omega}_{g, n}^{\mathbb{P}^{1}}$, respectively.

Notice that no positive power of $\lambda$ appears in the TQFT (3.2) and in $\widetilde{R}$. So by the reconstruction, which also holds for $\widetilde{\Omega}_{g, n}^{\mathbb{P}^{1}}$ in the Chow ring, the base ring for the CohFT $\widetilde{\Omega}_{g, n}^{\mathbb{P}^{1}}$ can be taken to be the intersection

$$
\mathbb{Q}\left[\lambda^{ \pm}, \phi\right] \cap \mathbb{Q}\left[\lambda^{-1}, \phi^{ \pm}\right]=\mathbb{Q}\left[\lambda^{-1}, \phi\right]
$$

We define a new CohFT $\Omega_{g, n}$ on the module $\mathbb{Q}[\phi]\langle 1, \widetilde{H}\rangle$ over the base ring $\mathbb{Q}[\phi]$ by setting $\lambda^{-1}=0$ in $\widetilde{\Omega}_{g, n}^{\mathbb{P}^{1}}$. Reconstruction in the Chow ring for $\widetilde{\Omega}_{g, n}^{\mathbb{P}^{1}}$ implies reconstruction in the Chow ring for $\Omega_{g, n}$.

The CohFT $\Omega_{g, n}$ has the same underlying TQFT (3.2) as before but the simpler $R$-matrix

$$
\left(\begin{array}{cc}
F_{1}^{e} & \sqrt{\phi} F_{1}^{o} \\
\frac{1}{\sqrt{\phi}} F_{0}^{o} & F_{0}^{e}
\end{array}\right)\left(-\frac{z}{6 \phi^{3 / 2}}, 0\right)=\left(\begin{array}{cc}
F_{1}^{e} & -\sqrt{\phi} F_{1}^{o} \\
-\frac{1}{\sqrt{\phi}} F_{0}^{o} & F_{0}^{e}
\end{array}\right)\left(\frac{z}{6 \phi^{3 / 2}}, 0\right) .
$$




\section{F. JANDA}

TABLE 1. Degree rules

\begin{tabular}{c|c|c|c|c|} 
& $\lambda$ & $q$ & $\phi$ & $C \in A^{i}\left(\bar{M}_{g, n}\right)$ \\
\hline$\Omega_{g, n}^{\mathbb{P}^{1}}\left(\widetilde{H}^{a_{1}}, \ldots, \widetilde{H}^{a_{n}}\right)$ & 1 & 2 & 2 & $i$ \\
$\widetilde{\Omega}_{g, n}^{\mathbb{P}^{1}}\left(\widetilde{H}^{a_{1}}, \ldots, \widetilde{H}^{a_{n}}\right)$ & 1 & 2 & 2 & $3 i$ \\
$\Omega_{g, n}\left(\widetilde{H}^{a_{1}}, \ldots, \widetilde{H}^{a_{n}}\right)$ & & & 2 & $3 i$
\end{tabular}

From their definitions, we can make the $F_{i}(z, 0)$ explicit:

$$
\begin{aligned}
& F_{0}(z, 0) \asymp \frac{1}{\sqrt{2 \pi}} \int_{-\infty}^{\infty} e^{-\frac{x^{2}}{2}} e^{-\frac{x^{3}}{6} \sqrt{-z}} \mathrm{~d} x \asymp \sum_{i=0}^{\infty} \frac{(6 i-1) ! !}{(2 i) !} \frac{(-z)^{i}}{36^{i}}=A(6 z), \\
& F_{1}(z, 0) \asymp \frac{1}{\sqrt{2 \pi}} \int_{-\infty}^{\infty} e^{-\frac{x^{2}}{2}} e^{-\frac{x^{3}}{6} \sqrt{-z}}(1+x \sqrt{-z}) \mathrm{d} x \asymp \cdots=B(6 z) .
\end{aligned}
$$

Here, the Faber-Zagier $A$ - and $B$-series (1.2) appear. Using the symplectic condition (3.4), we can also compute the inverse of the $R$-matrix to be equal to

$$
\left(\begin{array}{cc}
A^{e} & \sqrt{\phi} B^{o} \\
\frac{1}{\sqrt{\phi}} A^{o} & B^{e}
\end{array}\right)\left(\begin{array}{c}
z \\
\phi^{3 / 2}
\end{array}\right),
$$

where $A^{e}$ and $B^{e}$ are the even parts of $A$ and $B$, respectively, and similarly $A^{o}$ and $B^{o}$ denote the odd parts. The coefficients of the inverse $R$-matrix are elements of $\mathbb{Q}\left[\phi^{ \pm}\right] \llbracket q \rrbracket$.

In cohomology, the CohFT of the shifted Witten's 3-spin class as described in [PPZ15] coincides with $\Omega_{g, n}$ if we identify the parameters $\phi$ here and in [PPZ15] as well as the bases $\{1, \widetilde{H}\}$ and $\left\{\partial_{x}, \partial_{y}\right\}$. This is because the TQFT (3.2) (compare to [PPZ15, Lemma 3.3]) and inverse $R$ matrix (3.5) (compare to [PPZ15, equation (14)]) completely agree. ${ }^{1}$ It is not clear whether $\Omega_{g, n}$ agrees with Witten's 3-spin class in the Chow ring since it is not known whether reconstruction in the Chow ring holds for the 3-spin theory.

\subsection{Relations}

Recall from the previous section that there exists a CohFT $\Omega_{g, n}$ defined on the $\mathbb{Q}[\phi]$-module $\mathbb{Q}[\phi]\langle 1, \widetilde{H}\rangle$ which coincides with the CohFT of shifted Witten's 3-spin class in cohomology but for which reconstruction holds in the Chow ring. Let us fix a choice of $a_{1}, \ldots, a_{n} \in\{0,1\}$ for this section.

The fact that the inverse $R$-matrix (3.5) has poles in $\phi$ implies the existence of tautological relations: The reconstruction formula (2.3) for the CohFT $\Omega_{g, n}$ defines an element

$$
\mathcal{R}_{g, n}\left(\widetilde{H}^{a_{1}}, \ldots, \widetilde{H}^{a_{n}}\right) \in \mathcal{S}_{g, n} \otimes \mathbb{Q}\left[\phi^{ \pm}\right],
$$

projecting to $\Omega_{g, n}\left(\widetilde{H}^{a_{1}}, \ldots, \widetilde{H}^{a_{n}}\right)$ under $q: \mathcal{S}_{g, n} \rightarrow R^{*}\left(\bar{M}_{g, n}\right)$. So all coefficients of $\phi^{c}$ in $\mathcal{R}_{g, n}$ for $c<0$ have to project to zero and thus are tautological relations. As we will see in this section, these relations turn out to be nontrivial and are in fact enough to prove Theorem 1.1.

\footnotetext{
${ }^{1}$ Note that the TQFT and inverse $R$-matrix in [PPZ15] are written in the rescaled basis $\left\{\hat{\partial}_{x}, \hat{\partial}_{y}\right\}$, where $\hat{\partial}_{x}=\phi^{1 / 4} \partial_{x}$ and $\hat{\partial}_{y}=\phi^{-1 / 4} \partial_{y}$.
} 


\section{Relations on $\bar{M}_{g, n}$ Via Equivariant Gromov-Witten theory of $\mathbb{P}^{1}$}

We can characterize the part of $\mathcal{R}_{g, n}$ with poles in $\phi$ in a different way: By the dimension analysis from Section 3.1, the coefficient of $\lambda^{j} q^{d}$ in $\Omega_{g, n}^{\mathbb{P} 1}\left(\widetilde{H}^{a_{1}}, \ldots, \widetilde{H}^{a_{n}}\right)$ is an element of $A^{k}\left(\bar{M}_{g, n}\right)$ for

$$
k=g-1-2 d-j+\sum_{i=1}^{n} a_{i} .
$$

Hence, $\Omega_{g, n}^{\mathbb{P}^{1}}\left(\widetilde{H}^{a_{1}}, \ldots, \widetilde{H}^{a_{n}}\right)$ is homogeneous of degree

$$
g-1+\sum_{i=1}^{n} a_{i}
$$

if we assign degrees according to the first row of Table 1.

Recall from Section 3.4 that $\widetilde{\Omega}_{g, n}^{\mathbb{P}^{1}}=\varphi \circ \Omega_{g, n}^{\mathbb{P}^{1}}$, where $\varphi$ replaces each $C \in A^{i}\left(\bar{M}_{g, n}\right)$ by a constant multiple of $C \lambda^{-2 i}$. Therefore $\widetilde{\Omega}_{g, n}^{\mathbb{P}^{1}}\left(\widetilde{H}^{a_{1}}, \ldots, \widetilde{H}^{a_{n}}\right)$ is also homogeneous of degree (3.6) if we assign degrees as in the second row of Table 1 . Finally, $\Omega_{g, n}\left(\widetilde{H}^{a_{1}}, \ldots, \widetilde{H}^{a_{n}}\right)$ is homogeneous of the same degree (3.6) if we assign degrees as in the third row of Table 1.

The conclusion is that the part of

$$
\mathcal{R}_{g, n}\left(\widetilde{H}^{a_{1}}, \ldots, \widetilde{H}^{a_{n}}\right)
$$

with poles in $\phi$ corresponds to the part whose degree in the Chow ring is greater than

$$
\frac{1}{3}\left(g-1+\sum_{i=1}^{n} a_{i}\right) .
$$

This is exactly the degree of Witten's 3-spin class. In fact, as discussed before, in cohomology the CohFT $\Omega_{g, n}$ is the same as the CohFT of Witten's 3-spin class and, as in [PPZ15], the considered relations are those of degree greater than the degree (3.8) of Witten's class. So the relations we consider are the same elements of the strata algebra as in [PPZ15]. Starting from the fact that the Givental-Teleman classification of semisimple CohFTs holds in the Chow ring for the CohFT $\Omega_{g, n}^{\mathbb{P}^{1}}$, we have shown that the relations considered in [PPZ15] hold not only in cohomology but also in Chow rings.

In [PPZ15], the resulting relations are formally simplified to give the relations of Theorem 1.1. Thus, we can conclude the proof of Theorem 1.1 here. For the convenience of the reader we provide a summary of the arguments below.

\subsection{Computing the relations}

Following [PPZ15], we indicate how the relations from the vanishing of (3.7) in degree greater than (3.8) imply the relations of Theorem 1.1. For simplicity, let us set $\phi=1$. We expand the reconstruction formula (2.3) defining (3.7) using the explicit inverse $R$-matrix (3.5) and the formula (3.2) for the TQFT. The terms which give a relation are those of degree greater than (3.8), which explains inequality (1.4). For a dual graph $\Gamma$, the powers of 2 in (3.2) yield

$$
\prod_{v} 2^{g(v)}=2^{g} 2^{-h^{1}(\Gamma)}
$$


The edge term in the reconstruction formula is

$$
\begin{aligned}
\frac{R^{-1}\left(\psi^{\prime}\right) \otimes R^{-1}\left(\psi^{\prime \prime}\right)-\operatorname{Id} \otimes \operatorname{Id}}{-\psi^{\prime}-\psi^{\prime \prime}} \eta^{-1} & \frac{1 \otimes \widetilde{H}+\widetilde{H} \otimes 1-\left(A^{e}\left(\psi^{\prime}\right) 1+A^{o}\left(\psi^{\prime}\right) \widetilde{H}\right) \otimes\left(B^{o}\left(\psi^{\prime \prime}\right) 1+B^{e}\left(\psi^{\prime \prime}\right) \widetilde{H}\right)}{\psi^{\prime}+\psi^{\prime \prime}} \\
= & -\frac{\left(B^{o}\left(\psi^{\prime}\right) 1+B^{e}\left(\psi^{\prime}\right) \widetilde{H}\right) \otimes\left(A^{e}\left(\psi^{\prime \prime}\right) 1+A^{o}\left(\psi^{\prime \prime}\right) \widetilde{H}\right)}{\psi^{\prime}+\psi^{\prime \prime}} \\
& =\left[\Delta_{e}\right]_{\zeta^{\prime 0} \zeta^{\prime \prime \prime}}(1 \otimes 1)+\left[\Delta_{e}\right]_{\zeta^{\prime 1} \zeta^{\prime \prime \prime}}(\widetilde{H} \otimes 1)+\left[\Delta_{e}\right]_{\zeta^{\prime 0} \zeta^{\prime \prime 1}}(1 \otimes \widetilde{H})+\left[\Delta_{e}\right]_{\zeta^{\prime 1} \zeta^{\prime \prime 1}}(\widetilde{H} \otimes \widetilde{H}),
\end{aligned}
$$

where $\left[\Delta_{e}\right]_{\zeta^{\prime i} \zeta^{\prime \prime j}}$ is the coefficient of $\zeta^{\prime i} \zeta^{\prime \prime j}$ in the edge series $\Delta_{e}$ from the introduction. Similarly, in the reconstruction formula, the contribution of the $i$ th marking and of an extra marking can be expressed in terms of $B_{i}$ and $\kappa_{v}$, respectively. As necessitated by the case distinction in (3.2), we can keep track of the parity of the number of $\widetilde{H}$-insertions into the TQFT at vertex $v$ using an additional variable $\zeta_{v}$. By careful inspection, we see that (3.7) at $\phi=1$ coincides with (1.3) times $2^{g}$. We have thus arrived at the statement of Theorem 1.1.

\section{Appendix. Virtual localization for projective spaces (after Givental)}

In this appendix, we recall Givental's localization calculation [Giv01b] (see also [LP04] for a more leisurely treatment), which proves that the CohFT from equivariant $\mathbb{P}^{m}$ can be obtained from the trivial theory via a specific $R$-matrix action. We first recall localization in the space of stable maps to $\mathbb{P}^{m}$, in Section A.1. Next, in Section A.2, we group the localization contributions according to the dual graph of the source curve. We collect identities following from the string and dilaton equation in Section A.3, before applying them to finish the computation in Section A.4. In Section A.5, we collect properties of the $R$-matrix. Finally, in Section A.6, we give Givental's mirror description [Giv01a] of the $R$-matrix.

\section{A.1 Localization in the space of stable maps}

Let $T=\left(\mathbb{C}^{*}\right)^{m+1}$ act diagonally on $\mathbb{P}^{m}$. The equivariant Chow rings of a point pt and $\mathbb{P}^{m}$ are given by

$$
A_{T}^{*}(\mathrm{pt}) \cong \mathbb{Q}\left[\lambda_{0}, \ldots, \lambda_{m}\right], \quad A_{T}^{*}\left(\mathbb{P}^{m}\right) \cong \mathbb{Q}\left[H, \lambda_{0}, \ldots, \lambda_{m}\right] / \prod_{i=0}^{m}\left(H-\lambda_{i}\right),
$$

where $H$ is a lift of the hyperplane class. Furthermore, let $\eta$ be the equivariant Poincaré pairing.

There are $m+1$ fixed points $p_{0}, \ldots, p_{m}$ for the $T$-action on $\mathbb{P}^{m}$. The characters of the action of $T$ on the tangent space $T_{p_{i}} \mathbb{P}^{m}$ are given by $\lambda_{i}-\lambda_{j}$ for $j \neq i$. Hence the corresponding equivariant Euler class $e_{i}$ is

$$
e_{i}=\prod_{j \neq i}\left(\lambda_{i}-\lambda_{j}\right)
$$

The equivariant class $e_{i}$ also serves as the inverse of the norms of the equivariant (classical) idempotents:

$$
\phi_{i}=e_{i}^{-1} \prod_{j \neq i}\left(H-\lambda_{j}\right)
$$

The virtual localization formula [GP99] implies that the virtual fundamental class can be 


\section{Relations on $\bar{M}_{g, n}$ Via equivariant Gromov-Witten theory of $\mathbb{P}^{1}$}

split into a sum

$$
\left[\bar{M}_{g, n}\left(\mathbb{P}^{m}, d\right)\right]_{T}^{\mathrm{vir}}=\sum_{X} \iota_{X, *} \frac{[X]_{T}^{\mathrm{vir}}}{e_{T}\left(N_{X, T}^{\mathrm{vir}}\right)}
$$

of contributions of fixed loci $X$. Here, $N_{X, T}^{\mathrm{vir}}$ denotes the virtual normal bundle of $X$ in $\bar{M}_{g, n}\left(\mathbb{P}^{m}, d\right)$ and $e_{T}$ the equivariant Euler class. Because of the denominator, the fixed-point contributions are defined only after localizing by the elements $\lambda_{0}, \ldots, \lambda_{m}$. By studying the $\mathbb{C}^{*}$-action on deformations and obstructions of stable maps, $e_{T}\left(N_{X, T}^{\mathrm{vir}}\right)$ can be computed explicitly.

The fixed loci can be labeled by certain decorated graphs. These consist of

- a graph $(V, E)$,

- an assignment $\zeta: V \rightarrow\left\{p_{0}, \ldots, p_{m}\right\}$ of fixed points,

- a genus assignment $g: V \rightarrow \mathbb{Z}_{\geqslant 0}$,

- a degree assignment $d: E \rightarrow \mathbb{Z}_{>0}$,

- an assignment $p:\{1, \ldots n\} \rightarrow V$ of marked points,

such that the graph is connected and contains no self-edges, two adjacent vertices are not assigned to the same fixed point and we have

$$
g=h^{1}(\Gamma)+\sum_{v \in V} g(v), \quad d=\sum_{e \in E} d(e) .
$$

A vertex $v \in V$ is called stable if $2 g(v)-2+n(v)>0$, where $n(v)$ is the number of outgoing edges at $v$.

The fixed locus corresponding to a graph is characterized by the conditions that stable vertices $v \in V$ of the graph correspond to contracted genus $g(v)$ components of the domain curve and that edges $e \in E$ correspond to multiple covers of degree $d(e)$ of the torus fixed line between two fixed points. An unstable vertex $v$ with $(g(v), n(v))=(0,2)$ corresponds to either a node connecting two multiple covers of a line or a marking at the end of one of the multiple covers. When $(g(v), n(v))=(0,2)$, the vertex $v$ corresponds to an unmarked point at the end of a multiple cover. Such a fixed locus is isomorphic to a product of moduli spaces of curves

$$
\prod_{v \in V} \bar{M}_{g(v), n(v)}
$$

up to a finite map.

For a fixed locus $X$ corresponding to a given graph, the Euler class $e_{T}\left(N_{X, T}^{\mathrm{vir}}\right)$ is a product of factors corresponding to the geometry of the graph,

$$
e_{T}\left(N_{X, T}^{\mathrm{vir}}\right)=\prod_{v, \text { stable }} \frac{e\left(\mathbb{E}^{*} \otimes T_{\mathbb{P} m}, \zeta(v)\right.}{e_{\zeta(v)}} \prod_{\text {nodes }} \frac{e_{\zeta}}{-\psi_{1}-\psi_{2}} \prod_{\substack{g(v)=0 \\ n(v)=1}}\left(-\psi_{v}\right) \prod_{e} \operatorname{Contr}_{e} .
$$

In the first product, $\mathbb{E}^{*}$ denotes the dual of the Hodge bundle, $T_{\mathbb{P}^{m}, \zeta(v)}$ is the tangent space of $\mathbb{P}^{m}$ at $\zeta(v)$, and all bundles and Euler classes should be considered equivariantly. The second product is over nodes forced onto the domain curve by the graph. They correspond to stable vertices together with an outgoing edge, or vertices $v$ of genus 0 with $n(v)=2$. By $\psi_{1}$ and $\psi_{2}$, we denote the (equivariant) cotangent line classes at the two sides of the node. For example, the equivariant cotangent line class $\psi$ at a fixed point $p_{i}$ on a line mapped with degree $d$ to a fixed 
line is more explicitly given by

$$
-\psi=\frac{\lambda_{i}-\lambda_{j}}{d}
$$

where $p_{j}$ is the other fixed point on the fixed line. The explicit expressions for the last two factors of (A.1) can be found in [GP99], but will play no role for us. It is only important that they depend only on local data.

\section{A.2 General procedure}

We set $W$ to be $A_{T}^{*}\left(\mathbb{P}^{m}\right)$ with all equivariant parameters localized. For $v_{1}, \ldots, v_{n} \in W$, the (full) CohFT $\Omega_{g, n}$ from equivariant $\mathbb{P}^{m}$ is defined by

$$
\Omega_{g, n}^{t}\left(v_{1}, \ldots, v_{n}\right)=\sum_{d, k=0}^{\infty} \frac{q^{d}}{k !} \pi_{*} p_{*}\left(\prod_{i=1}^{n} \operatorname{ev}_{i}^{*}\left(v_{i}\right) \prod_{i=n+1}^{n+k} \operatorname{ev}_{i}^{*}(t) \cap\left[\bar{M}_{g, n+k}\left(\mathbb{P}^{m}, d\right)\right]^{\mathrm{vir}}\right),
$$

where

$$
t=t_{0} \phi_{0}+\cdots+t_{m} \phi_{m}
$$

is a formal point on $W$, the map $\pi$ forgets the last $k$ markings and $p$ forgets the map. We want to calculate the push-forward via virtual localization. In the end, we will arrive at the formula of the $R$-matrix action as described in Section 2.3 for an endomorphism-valued power series $R_{\mathbb{P} m}(z)$. In the following, we will systematically suppress the dependence on $t$ in the notation. In the main part of this paper, we are interested in the case where $t$ is set to zero.

For the localization computation, we start by remarking that for each localization graph for (A.2), there exists a dual graph of $\bar{M}_{g, n}$ corresponding to the topological type of the stabilization under $\pi p$ of a generic source curve of that locus. What gets contracted under the stabilization maps are trees of rational curves. There are three types of these unstable trees of rational curves:

(i) Those which contain one of the $n$ markings and are connected to a stable component.

(ii) Those which are connected to two stable components and contain none of the $n$ markings.

(iii) Those which are connected to one stable component but contain none of the $n$ markings.

Here, a stable component is a component of the source curve not contracted by the stabilization for $\pi p$. The first two types of trees correspond, respectively, to the preimage under the stabilization of

(i) one of the $n$ markings,

(ii) one of the nodes.

Each type of tree gives rise to a series of localization contributions, and we want to record it using the fact that the same contributions already occur in genus zero.

Let $W^{\prime}$ be an abstract free module over the same base ring as $W$ with a basis $w_{0}, \ldots, w_{m}$ labeled by the fixed points of the $T$-action on $\mathbb{P}^{m}$. We will later identify $W^{\prime}$ with $W$ in a nontrivial way (see equation (A.11)). The type (i) contributions are recorded by

$$
\widetilde{R}^{-1}=\sum_{i} \widetilde{R}_{i}^{-1} w_{i} \in \operatorname{Hom}\left(W, W^{\prime}\right) \llbracket z \rrbracket,
$$




\section{Relations on $\bar{M}_{g, n}$ Via Equivariant Gromov-Witten theory of $\mathbb{P}^{1}$}

the homomorphism-valued power series such that

$$
\widetilde{R}_{i}^{-1}(v)=\eta\left(e_{i} \phi_{i}, v\right)+\sum_{d, k=0}^{\infty} \frac{q^{d}}{k !} \sum_{\Gamma \in G_{d, k, i}^{1}} \frac{1}{\operatorname{Aut}(\Gamma)} \operatorname{Contr}_{\Gamma}(v),
$$

where $G_{d, k, i}^{1}$ is the set of localization graphs for $\bar{M}_{0,2+k}\left(\mathbb{P}^{m}, d\right)$ such that the first marking is at a valence 2 vertex at fixed point $i$ and $\operatorname{Contr}_{\Gamma}(v)$ is the contribution for graph $\Gamma$ for the integral

$$
\int_{\bar{M}_{0,2+k}\left(\mathbb{P}^{m}, d\right)} \frac{e_{i}}{-z-\psi_{1}} \mathrm{ev}_{2}^{*}(v) \prod_{l=3}^{2+k} \mathrm{ev}_{l}^{*}(t) .
$$

We define the integral in the case $(d, k)=(0,0)$ to be zero and will do likewise for other integrals over nonexisting moduli spaces.

The type (ii) contributions are recorded by the bivector

$$
\widetilde{V}=\sum_{i} \widetilde{V}^{i j} w_{i} \otimes w_{j} \in W^{\prime \otimes 2} \llbracket z, w \rrbracket
$$

which is defined by

$$
\widetilde{V}^{i j}=\sum_{d, k=0}^{\infty} \frac{q^{d}}{k !} \sum_{\Gamma \in G_{d, k, i, j}^{2}} \frac{1}{\operatorname{Aut}(\Gamma)} \operatorname{Contr}_{\Gamma},
$$

where $G_{d, k, i, j}^{2}$ is the set of localization graphs for $\bar{M}_{0,2+k}\left(\mathbb{P}^{m}, d\right)$ such that the first and second marking are at valence 2 vertices at fixed points $i$ and $j$, respectively, and $\operatorname{Contr}_{\Gamma}$ is the contribution for graph $\Gamma$ for the integral

$$
\int_{\bar{M}_{0,2+k}\left(\mathbb{P}^{m}, d\right)} \frac{e_{i} e_{j}}{\left(-z-\psi_{1}\right)\left(-w-\psi_{2}\right)} \prod_{l=3}^{2+k} \mathrm{ev}_{l}^{*}(t) .
$$

Finally, the type (iii) contribution is a vector

$$
\widetilde{T}=\sum_{i} \widetilde{T}_{i} w_{i} \in W^{\prime} \llbracket z \rrbracket
$$

which is defined by

$$
\widetilde{T}_{i}=t+\sum_{d, k=0}^{\infty} \frac{q^{d}}{k !} \sum_{\Gamma \in G_{d, k, i}^{3}} \frac{1}{\operatorname{Aut}(\Gamma)} \operatorname{Contr}_{\Gamma},
$$

where $G_{d, k, i}^{3}$ is the set of localization graphs for $\bar{M}_{0,1+k}\left(\mathbb{P}^{m}, d\right)$ such that the first marking is at a valence 2 vertex at fixed point $i$ and $\operatorname{Contr}_{\Gamma}(v)$ is the contribution for graph $\Gamma$ for the integral

$$
\int_{\bar{M}_{0,1+k}\left(\mathbb{P}^{m}, d\right)} \frac{e_{i}}{-z-\psi} \prod_{l=2}^{1+k} \mathrm{ev}_{l}^{*}(t) .
$$

With these contributions, we can write the CohFT already in a form quite similar to the $R$-matrix action formula (2.3). Let $\omega_{g, n}$ be the $n$-form on $W^{\prime}$ which vanishes if $w_{i}$ and $w_{j}$ for $i \neq j$ are inputs, which satisfies

$$
\omega_{g, n}\left(w_{i}, \ldots, w_{i}\right)=\frac{e\left(\mathbb{E}^{*} \otimes T_{\mathbb{P}^{m}, p_{i}}\right)}{e_{i}}=e_{i}^{g-1} \prod_{j \neq i} c_{\lambda_{j}-\lambda_{i}}(\mathbb{E})
$$


and which for $n=0$ is defined similarly as in Example 2.5. We have

$$
\Omega_{g, n}^{t}\left(v_{1}, \ldots, v_{n}\right)=\sum_{\Gamma} \frac{1}{\operatorname{Aut}(\Gamma)} \xi_{*}\left(\prod_{v} \sum_{k=0}^{\infty} \frac{1}{k !} \pi_{*} \omega_{g_{v}, n_{v}+k}(\ldots)\right),
$$

where we put

(i) $\widetilde{R}^{-1}(\psi)\left(v_{i}\right)$ into the argument corresponding to marking $i$,

(ii) one half of $\widetilde{V}\left(\psi_{1}, \psi_{2}\right)$ into an argument corresponding to a node and

(iii) $\widetilde{T}(\psi)$ into all additional arguments.

We will still need to apply the string and dilaton equation in order to make $\widetilde{T}(\psi)$ a multiple of $\psi^{2}$, like the corresponding series in the reconstruction, express the Hodge classes via Mumford's formula and then relate the series to the $R$-matrix.

\section{A.3 String and dilaton equation}

We want to use the string and dilaton equation to bring a series

$$
\sum_{k=0}^{\infty} \frac{1}{k !} \pi_{*}\left(\prod_{i=1}^{n} \frac{1}{-x_{i}-\psi_{i}} \prod_{i=n+1}^{n+k} Q\left(\psi_{i}\right)\right)
$$

where $\pi: \bar{M}_{g, n+k} \rightarrow \bar{M}_{g, n}$ is the forgetful map and $Q=Q_{0}+z Q_{1}+z^{2} Q_{2}+\cdots$ is a formal series, into a canonical form.

By the string equation, (A.4) is annihilated by

$$
\mathcal{L}^{\prime}=\mathcal{L}+\sum_{i=1}^{n} \frac{1}{x_{i}}
$$

where $\mathcal{L}$ is the string operator

$$
\mathcal{L}=\frac{\partial}{\partial Q_{0}}-Q_{1} \frac{\partial}{\partial Q_{0}}-Q_{2} \frac{\partial}{\partial Q_{1}}-Q_{3} \frac{\partial}{\partial Q_{2}}-\cdots
$$

Moving along the string flow for some time $-u$, that is, applying $\left.e^{t \mathcal{L}^{\prime}}\right|_{t=-u}$ to (A.4), gives

$$
\sum_{k=0}^{\infty} \frac{1}{k !} \pi_{*}\left(\prod_{i=1}^{n} \frac{e^{-\frac{u}{x_{i}}}}{-x_{i}-\psi_{i}} \prod_{i=n+1}^{n+k} Q^{\prime}\left(\psi_{i}\right)\right)
$$

for a new formal series $Q^{\prime}=Q_{0}^{\prime}+z Q_{1}^{\prime}+z^{2} Q_{2}^{\prime}+\cdots$. In the case that

$$
u=\sum_{k=1}^{\infty} \frac{1}{k !} \int_{M_{0,2+k}} \prod_{i=3}^{2+k} Q\left(\psi_{i}\right)
$$

which we will assume from now on, the new series $Q^{\prime}$ satisfies $Q_{0}^{\prime}=0$. This is because the string equation implies $\mathcal{L} u=1$ and therefore applying $\left.e^{t \mathcal{L}}\right|_{t=-u}$ to $u$ gives, on the one hand, $u-\left.t\right|_{t=-u}=0$ and, on the other hand, the definition of $u$ with $Q$ replaced by $Q^{\prime}$, which for dimension reasons is a nonzero multiple of $Q_{0}^{\prime}$.

Next, by applying the dilaton equation, we can remove the linear part from the series $Q_{0}^{\prime}$ :

$$
\sum_{k=0}^{\infty} \frac{1}{k !} \pi_{*}\left(\prod_{i=1}^{n} \frac{1}{-x_{i}-\psi_{i}} \prod_{i=n+1}^{n+k} Q\left(\psi_{i}\right)\right)=\sum_{k=0}^{\infty} \frac{\Delta^{\frac{2 g-2+n+k}{2}}}{k !} \pi_{*}\left(\prod_{i=1}^{n} \frac{e^{-\frac{u}{x_{i}}}}{-x_{i}-\psi_{i}} \prod_{i=n+1}^{n+k} Q^{\prime \prime}\left(\psi_{i}\right)\right)
$$


where $Q^{\prime \prime}=Q^{\prime}-Q_{1}^{\prime} z$ and

$$
\Delta^{\frac{1}{2}}=\left(1-Q_{1}^{\prime}\right)^{-1}=\sum_{k=0}^{\infty} \frac{1}{k !} \int_{M_{0,3+k}} \prod_{i=4}^{3+k} Q\left(\psi_{i}\right) .
$$

We will also need identities in the degenerate cases $(g, n)=(0,2)$ and $(g, n)=(0,1)$. In the first case, there is the identity

$$
\frac{1}{-z-w}+\sum_{k=1}^{\infty} \frac{1}{k !} \int_{M_{0,2+k}} \frac{1}{-z-\psi_{1}} \frac{1}{-w-\psi_{2}} \prod_{i=3}^{2+k} Q\left(\psi_{i}\right)=\frac{e^{-u / z+-u / w}}{-z-w} .
$$

In order to see that (A.6) is true, we use that the left-hand side is annihilated by $\mathcal{L}+1 / z+1 / w$ in order to move from $Q$ to $Q^{\prime}$ via the string flow and notice that there, all the integrals vanish for dimension reasons. Similarly, there is the identity

$$
1-\frac{Q(z)}{z}-\frac{1}{z} \sum_{k=2}^{\infty} \frac{1}{k !} \int_{M_{0,1+k}} \prod_{i=2}^{1+k} Q\left(\psi_{i}\right)=e^{-u / z}\left(1-\frac{Q^{\prime}(z)}{z}\right)=e^{-u / z}\left(\Delta^{-\frac{1}{2}}-\frac{Q^{\prime \prime}(z)}{z}\right),
$$

which can be proven like the previous identity by using that the left-hand side is annihilated by $\mathcal{L}+1 / z$.

We define the functions $u_{i}$ and $\left(\Delta_{i} / e_{i}\right)^{1 / 2}$ for $i \in\{0, \ldots, m\}$ to be the $u$ and $\Delta^{1 / 2}$ at the points $Q=\widetilde{T}_{i}$ from the previous section.

\section{A.4 Computation of the localization series}

We apply (A.5) to (A.3) and obtain

$$
\Omega_{g, n}^{t}\left(v_{1}, \ldots, v_{n}\right)=\sum_{\Gamma} \frac{1}{\operatorname{Aut}(\Gamma)} \xi_{*}\left(\prod_{v} \sum_{k=0}^{\infty} \frac{1}{k !} \pi_{*} \omega_{g_{v}, n_{v}+k}^{\prime}(\ldots)\right),
$$

where we put

(i) $R^{-1}(\psi)\left(v_{i}\right)$ into the argument corresponding to marking $i$,

(ii) one half of $V\left(\psi_{1}, \psi_{2}\right)$ into an argument corresponding to a node and

(iii) $T(\psi)$ into all additional arguments.

Here $R^{-1}, V$ and $T$ are defined exactly as $\widetilde{R}^{-1}, \widetilde{V}$ and $\widetilde{T}$ but with the replacement

$$
\frac{e_{i}}{-x-\psi} \rightsquigarrow \frac{e_{i} e^{-u_{i} / x}}{-x-\psi}
$$

made at the factors we put at the ends of the trees. The form $\omega_{g, n}^{\prime}$ satisfies

$$
\omega_{g, n}^{\prime}\left(w_{i}, \ldots, w_{i}\right)=\Delta_{i}^{(2 g-2+n) / 2} e_{i}^{-n / 2} \prod_{j \neq i} c_{\lambda_{j}-\lambda_{i}}(\mathbb{E}) .
$$

We now want to compute $R^{-1}, V$ and $T$ in terms of the homomorphism-valued series $S^{-1}(z) \in$ $\operatorname{Hom}\left(W, W^{\prime}\right) \llbracket z \rrbracket$ with $w_{i}$-component

$$
S_{i}^{-1}(z)=\left\langle\frac{e_{i} \phi_{i}}{-z-\psi},-\right\rangle:=\eta\left(e_{i} \phi_{i},-\right)+\sum_{d, k=0}^{\infty} \frac{q^{d}}{k !} \int_{\bar{M}_{0,2+k}\left(\mathbb{P}^{m}, d\right)} \frac{\mathrm{ev}_{1}^{*}\left(e_{i} \phi_{i}\right)}{-z-\psi_{1}} \mathrm{ev}_{2}^{*}(-) \prod_{j=3}^{k+2} \mathrm{ev}_{j}^{*}(t) .
$$




\section{F. JANDA}

We start by computing $S^{-1}$ via localization. Using that in genus zero, the Hodge bundle is trivial, we find that at the vertex with the first marking we need to compute integrals exactly as in (A.6), where the first summand stands for the case that the vertex is unstable and the second summand stands for the case that the vertex is stable with $k$ trees of type (iii) and one tree of type (i) attached to it. Applying (A.6), we obtain

$$
S_{i}^{-1}(z)=e^{-u_{i} / z} R_{i}^{-1}(z) .
$$

Using the shorthand notation

$$
\left\langle\frac{v_{1}}{x_{1}-\psi}, \frac{v_{2}}{x_{2}-\psi}, \frac{v_{3}}{x_{3}-\psi}\right\rangle:=\sum_{d, k=0}^{\infty} \frac{q^{d}}{k !} \int_{\bar{M}_{0,3+k}\left(\mathbb{P}^{m}, d\right)} \frac{\mathrm{ev}_{1}^{*} v_{1}}{x_{1}-\psi_{1}} \frac{\mathrm{ev}_{2}^{*} v_{2}}{x_{2}-\psi_{2}} \frac{\mathrm{ev}_{3}^{*} v_{3}}{x_{3}-\psi_{3}} \prod_{i=4}^{3+k} \mathrm{ev}_{i}^{*}(t)
$$

for genus zero Gromov-Witten invariants and applying the string equation, we can also write

$$
S_{i}^{-1}(z)=-\frac{1}{z}\left\langle\frac{e_{i} \phi_{i}}{-z-\psi}, \mathbf{1},-\right\rangle .
$$

By the identity axiom and Witten-Dijkgraaf-Verlinde-Verlinde equation, we have

$$
\left\langle\frac{e_{i} \phi_{i}}{-z-\psi}, \frac{e_{j} \phi_{j}}{-w-\psi}, \mathbf{1}\right\rangle=\left\langle\frac{e_{i} \phi_{i}}{-z-\psi}, \frac{e_{j} \phi_{j}}{-w-\psi}, \bullet\right\rangle\langle\bullet, \mathbf{1}, \mathbf{1}\rangle=\left\langle\frac{e_{i} \phi_{i}}{-z-\psi}, \mathbf{1}, \bullet\right\rangle\left\langle\bullet, \mathbf{1}, \frac{e_{j} \phi_{j}}{-w-\psi}\right\rangle,
$$

where in the latter two expressions the $\bullet$ should be filled with $\eta^{-1}$, so that

$$
\begin{aligned}
& \frac{S_{i}^{-1}(z) \otimes S_{j}^{-1}(w)}{-z-w} \eta^{-1} \\
& \quad=\frac{\eta\left(e_{i} \phi_{i}, e_{j} \phi_{j}\right)}{-z-w}+\sum_{d, k=0}^{\infty} \frac{q^{d}}{k !} \int_{\bar{M}_{0,2+k}\left(\mathbb{P}^{m}, d\right)} \frac{\mathrm{ev}_{1}^{*}\left(e_{i} \phi_{i}\right)}{-z-\psi_{1}} \frac{\mathrm{ev}_{2}^{*}\left(e_{j} \phi_{j}\right)}{-w-\psi_{2}} \prod_{l=3}^{k+2} \operatorname{ev}_{l}^{*}(t) .
\end{aligned}
$$

We compute the right-hand side via localization. There are two cases in the localization depending on whether the first and second markings are at the same vertex or at different ones. In the first case, we apply (A.6) at this common vertex and obtain the total contribution

$$
\frac{e_{i} \delta_{i j} e^{-u_{i} / z-u_{j} / w}}{-z-w},
$$

which includes the unstable summand. In the other case, we apply (A.6) at the two vertices and obtain

$$
e^{-u_{i} / z-u_{j} / w} V^{i j}(z, w)
$$

So all together,

$$
V^{i j}(z, w)=\frac{\left(R_{i}^{-1}(z) \otimes R_{j}^{-1}(w)\right) \eta^{-1}-e_{i} \delta_{i j}}{-z-w} .
$$

Finally, we express $T$ in terms of $R$ by computing

$$
S_{i}^{-1}(z) \mathbf{1}=1-\frac{t_{i}}{z}-\frac{1}{z} \sum_{d, k=0}^{\infty} \frac{q^{d}}{k !} \int_{\bar{M}_{0,1+k}\left(\mathbb{P}^{m}, d\right)} \frac{\mathrm{ev}_{1}^{*}\left(e_{i} \phi_{i}\right)}{-z-\psi_{1}} \prod_{j=2}^{1+k} \mathrm{ev}_{j}^{*}(t)
$$

via localization. Applying (A.7) at the first marking, we find that

$$
S_{i}^{-1}(z) \mathbf{1}=e^{-u_{i} / z}\left(\Delta_{i}^{-1 / 2} e_{i}^{1 / 2}-\frac{T_{i}(z)}{z}\right) .
$$




\section{Relations on $\bar{M}_{g, n}$ Via Equivariant Gromov-Witten theory of $\mathbb{P}^{1}$}

So

$$
T(z)=z\left(\sum_{i} \Delta_{i}^{-1 / 2} e_{i}^{1 / 2} w_{i}-R^{-1}(z) \mathbf{1}\right) .
$$

By (A.8), the underlying TQFT of $\Omega_{g, 0}^{t}$ is given by $\sum_{i} \Delta_{i}^{g-1}$. This implies that the $\Delta_{i}$ need to be the inverses of the norms of the idempotents for the quantum product of equivariant $\mathbb{P}^{m}$ (because these are pairwise different). Since $\widetilde{T}$ vanishes at $(t, q)=0$, we have that $\Delta_{i}$ agrees with $e_{i}$ at $(t, q)=0$. Therefore, we can identify $W^{\prime}$ with $W$ via

$$
W^{\prime} \rightarrow W, \quad w_{i} \mapsto \sqrt{\Delta_{i} / e_{i}} \epsilon_{i},
$$

where $\epsilon_{i}$ is the idempotent element which coincides with $\phi_{i}$ at $(t, q)=0$. The previous results then say exactly that $\Omega^{t}$ is obtained from the CohFT $\omega^{\prime}$ by the action of the $R$-matrix $R$. In turn, Example 2.11 implies that $\omega^{\prime}$ is obtained from the TQFT by the action of an $R$-matrix $R_{\text {Mumford }}$ which is diagonal in the basis of idempotents and has entries

$$
\exp \left(\sum_{i=1}^{\infty} \frac{B_{2 i}}{2 i(2 i-1)} \sum_{j \neq i}\left(\frac{z}{\lambda_{j}-\lambda_{i}}\right)^{2 i-1}\right) .
$$

We define the endomorphism $R_{\mathbb{P} m}$ to be the composition

$$
R_{\mathbb{P} m}=R \cdot R_{\text {Mumford }} \cdot
$$

Therefore, the $R$-matrix action of $R_{\mathbb{P} m}$ takes the TQFT to $\Omega^{t}$.

\section{A.5 Characterization of $\boldsymbol{R}$-matrix}

We study the $R$-matrix $R_{\mathbb{P} m}$ defined in the previous section as the composition of $R$ and $R_{\text {Mumford }}$.

As we have seen, there is a power series $S^{-1}(z)$ in $z^{-1}$ strongly related to $R$. By considering (A.10) as $w+z \rightarrow 0$, we see that $S^{-1}(z)$ satisfies the symplectic condition; that is, its inverse $S(z)$ is the adjoint of $S^{-1}(-z)$ with respect to $\eta$. Using this, we can say, more explicitly, that the evaluation of $S(z)$ at the $i$ th normalized idempotent is the vector

$$
\left\langle\frac{\sqrt{e_{i}} \phi_{i}}{z-\psi}, \eta^{-1}\right\rangle
$$

In terms of $S(z)$, the relation between $R$ and $S$, when written in the basis of normalized idempotents, is

$$
R(z)=S(z) e^{-\operatorname{diag}\left(u_{0}, \ldots, u_{m}\right) / z} .
$$

The series $S(z)$ satisfies the quantum differential equation

$$
z \frac{\partial}{\partial t_{\mu}} S(z)=\phi_{\mu} \star S(z)
$$

for any $\mu$. This follows from the genus zero topological recursion relations

$$
z\left\langle\phi_{\mu}, \frac{\sqrt{e_{i}} \phi_{i}}{z-\psi}, \eta^{-1}\right\rangle=\left\langle\phi_{\mu}, \eta^{-1}, \bullet\right\rangle\left\langle\bullet, \frac{\sqrt{e_{i}} \phi_{i}}{z-\psi}\right\rangle,
$$

where, as previously, $\eta^{-1}$ should be inserted at the two

The divisor axiom in Gromov-Witten theory determines the $q$-dependence of $S$. We have

$$
\left\langle\frac{\sqrt{e_{i}} \phi_{i}}{z-\psi}, \eta^{-1}, H\right\rangle=\left\langle\frac{\sqrt{e_{i}} H \phi_{i}}{z(z-\psi)}, \eta^{-1}\right\rangle+d\left\langle\frac{\sqrt{e_{i}} \phi_{i}}{z-\psi}, \eta^{-1}\right\rangle=\left(\frac{\lambda_{i}}{z}+d\right)\left\langle\frac{\sqrt{e_{i}} \phi_{i}}{z-\psi}, \eta^{-1}\right\rangle
$$


and hence

$$
H \star S_{i}(z)=D S_{i}(z)+\lambda_{i} S_{i}(z)
$$

where

$$
D=z q \frac{\partial}{\partial q}
$$

The analog of equation (A.12) defines a new series $S_{\mathbb{P}^{1}}$. It satisfies the same symplectic condition, quantum differential equation and $q$-dependence (A.14) since $R_{\text {Mumford }}$ is diagonal and does not depend on the $t_{i}$.

We now consider the case $(t, q)=0$, which is also known as the classical limit. We have $\left.\Delta_{i}\right|_{(t, q)=0}=e_{i},\left.w_{i}\right|_{(t, q)=0}=\phi_{i}$ and $\left.\widetilde{R}\right|_{(t, q)=0}=\left.R\right|_{(t, q)=0}=$ Id. Therefore,

$$
\left.R_{\mathbb{P}^{1}}\right|_{(t, q)=0}=R_{\text {Mumford }}=\exp \left(\operatorname{diag}\left(b_{0}, \ldots, b_{m}\right)\right),
$$

where

$$
b_{j}=\sum_{i=1}^{\infty} \frac{B_{2 i}}{2 i(2 i-1)} \sum_{l \neq j}\left(\frac{z}{\lambda_{l}-\lambda_{j}}\right)^{2 i-1} .
$$

Proposition A.1. The series $R_{\mathbb{P}^{1}}(z)$ written in the basis of normalized idempotents is the unique matrix-valued power series in $z$ depending on parameters $\lambda_{0}, \ldots, \lambda_{m}, q$ and $t_{0}, \ldots, t_{m}$ such that there exist functions $u_{0}, \ldots, u_{m}$ independent of $z$ and a matrix-valued power series $S_{\mathbb{P}^{1}}$ in $z^{-1}$ such that

(i) the series $R_{\mathbb{P}^{1}}$ and $S_{\mathbb{P}^{1}}$ are related by (A.12),

(ii) $S_{\mathbb{P}^{1}}$ satisfies the quantum differential equation (A.13),

(iii) $S_{\mathbb{P}^{1}}$ satisfies (A.14) and

(iv) the classical limit $\left.R_{\mathbb{P}^{1}}\right|_{(t, q)=0}$ of $R$ is given by (A.15).

Proof. In this section, we have checked that $R_{\mathbb{P}^{1}}$ indeed satisfies these properties.

By a general analysis [Giv01a, Proposition 6.7] of the quantum differential equation, we see that the first two properties determine $R_{\mathbb{P}^{1}}$ up to functions constant in the $t_{i}$. Via (A.12), equation (A.14) also determines the dependence of $R_{\mathbb{P}^{1}}$ on $q$. Hence $R_{\mathbb{P}^{1}}$ is uniquely determined from its classical limit.

\section{A.6 Small equivariant mirror}

Following [Giv01a], we give a concrete description of the $R$-matrix for the equivariant GromovWitten theory of $\mathbb{P}^{m}$. In contrast to the previous sections of the appendix, we will soon restrict ourselves to the small quantum cohomology case $t=0$. To simplify the notation, we will leave out the subscript $\mathbb{P}^{m}$ in $R_{\mathbb{P}^{m}}$ and $S_{\mathbb{P}^{m}}$.

If we use alternative coordinates

$$
t=\widetilde{t}_{0}+\widetilde{t}_{1} H+\cdots+\widetilde{t}_{m} H^{m},
$$

the quantum differential equation implies

$$
z \frac{\partial}{\partial \widetilde{t}_{1}} S_{i}(z)=H \star S_{i}(z)=D S_{i}(z)+\lambda_{i} S_{i}(z) .
$$

So we can rewrite the differential equation (A.16) satisfied by $S_{i}$ to

$$
\left(z q \frac{\partial}{\partial q}+\lambda_{i}\right) S_{i}(z)=H \star S_{i}(z) .
$$




\section{Relations on $\bar{M}_{g, n}$ Via Equivariant Gromov-Witten theory of $\mathbb{P}^{1}$}

Now, it makes sense to restrict to $t=0$ and we will do so from now on (without changing the notation).

If we write $S_{\mu i}=\eta\left(S_{i}, \phi_{\mu}\right)$, we can rewrite (A.17) to

$$
D\left(S_{\mu i} e^{\ln (q) \lambda_{i} / z}\right)=S_{(\mu+1) i} e^{\ln (q) \lambda_{i} / z}
$$

for $\mu<m$ and

$$
\prod_{j=0}^{m}\left(D-\lambda_{j}\right)\left(S_{0 i} e^{\ln (q) \lambda_{i} / z}\right)=q S_{0 i} e^{\ln (q) \lambda_{i} / z}
$$

Proposition A.2. The series $R$ is the unique matrix-valued power series in $z$ depending on parameters $\lambda_{0}, \ldots, \lambda_{m}, q$ such that there exist functions $u_{0}, \ldots, u_{m}$ independent of $z$ and a matrix-valued power series $S$ in $z^{-1}$ such that

(i) the series $R$ and $S$ are related by (A.12),

(ii) $S$ satisfies the quantum differential equations (A.18) and (A.19),

(iii) the classical limit $\left.R\right|_{q=0}$ of $R$ is given by (A.15).

Proof. Equation (A.18) determines all of $S$ from $S_{0 i}$, which is determined from (A.19) up to additive integration constants depending on $\lambda_{0}, \ldots, \lambda_{m}$. Choosing the integration constants such that the classical limit condition holds determines $S$ up to constants (not depending on $\lambda_{0}, \ldots, \lambda_{m}$ ), which is enough to determine $R$ uniquely via (A.12).

Givental constructs asymptotic solutions $S_{0 i}$ to (A.19) via oscillating integrals on the mirror manifold

$$
\left\{\left(T_{0}, \ldots, T_{m}\right): e^{T_{0}} \cdots e^{T_{m}}=q\right\} \subset \mathbb{C}^{m+1}
$$

with superpotential

$$
F(T)=\sum_{j=0}^{m}\left(e^{T_{j}}+\lambda_{j} T_{j}\right)
$$

The integrals are given by

$$
S_{0 i} e^{\ln (q) \lambda_{i} / z}=(-2 \pi z)^{-m / 2} \int_{\Gamma_{i} \subset\left\{\sum T_{j}=\ln q\right\}} e^{F(T) / z} \omega
$$

along $m$-cycles $\Gamma_{i}$ through a specific critical point of the superpotential $F$ constructed using the Morse theory of the real part of $F / z$. The form $\omega$ is the restriction of $\mathrm{d} T_{0} \wedge \cdots \wedge \mathrm{d} T_{m}$. To see that the integrals are actual solutions, notice that applying $D-\lambda_{j}$ to the integral has the same effect as multiplying the integrand by $e^{T_{j}}$.

There are $m+1$ critical points at which it is possible to do a stationary phase expansion of $S_{0 i}$. Let us write $P_{i}$ for the solution to

$$
\prod_{i=0}^{m}\left(X-\lambda_{i}\right)=q
$$

with limit $\lambda_{i}$ as $q \rightarrow 0$. For each $i$, we need to choose the critical point $e^{T_{j}}=P_{i}-\lambda_{j}$ in order for 


\section{F. JANDA}

the factor $^{2}$

$$
\exp \left(u_{i} / z\right):=\exp \left(\left(\sum_{j=0}^{m}\left(P_{i}-\lambda_{j}+\lambda_{j} \ln \left(P_{i}-\lambda_{j}\right)\right)-\lambda_{i} \ln (q)\right) / z\right)
$$

of $S_{0 i}$ to be well defined in the limit as $q \rightarrow 0$. Shifting the integral to the critical point and scaling coordinates by $\sqrt{-z}$, we find

$$
S_{0 i}=e^{u_{i} / z} \int \exp \left(-\sum_{j}\left(P_{i}-\lambda_{j}\right) \sum_{k=3}^{\infty} \frac{T_{j}^{k}(-z)^{(k-2) / 2}}{k !}\right) \mathrm{d} \mu_{i}
$$

for the conditional Gaussian distribution

$$
\mathrm{d} \mu_{i}=(2 \pi)^{-m / 2} \exp \left(-\sum_{j}\left(P_{i}-\lambda_{j}\right) \frac{T_{j}^{2}}{2}\right) \omega .
$$

For the asymptotic expansion, we formally expand the exponential in (A.20) and integrate over the real part of the image of the mirror under the transformations we have performed. The integrals are moments of $\mu_{i}$ which can be computed using the covariance matrix

$$
\sigma_{i}\left(T_{k}, T_{l}\right)=\frac{1}{\Delta_{i}} \begin{cases}-\prod_{j \notin\{k, l\}}\left(P_{i}-\lambda_{j}\right) & \text { for } k \neq l, \\ \sum_{m \neq k} \prod_{j \notin\{k, m\}}\left(P_{i}-\lambda_{j}\right) & \text { for } k=l .\end{cases}
$$

Since odd moments of Gaussian distributions vanish, we find that the asymptotic expansion of $e^{-u_{i} / z} S_{0 i}$ is a power series in $z$. So, using (A.9) as a definition, by (A.18) the entries of the $R$-matrix in the basis of normalized idempotents are given by similar asymptotic expansion of

$$
\Delta_{k}^{-1 / 2} \prod_{j \neq k}\left(D+\lambda_{i}-P_{j}\right)\left(e^{-u_{i} / z} S_{0 i}\right) .
$$

We need to check that the $R$-matrix given in terms of asymptotics of oscillating integrals behaves correctly in the limit as $q \rightarrow 0$. By definition, in this limit, $P_{i} \rightarrow \lambda_{i}$. By symmetry, it is enough to consider the zeroth column. Set $x_{i}=e^{T_{i}}$. Then

$$
\begin{aligned}
& \lim _{q \rightarrow 0} R_{j 0} \asymp \lim _{q \rightarrow 0} e^{-u_{0} / z} \Delta_{0}^{-1 / 2} \prod_{k \neq j}\left(z q \frac{\mathrm{d}}{\mathrm{d} q}+\lambda_{j}-\lambda_{k}\right) S_{00}
\end{aligned}
$$

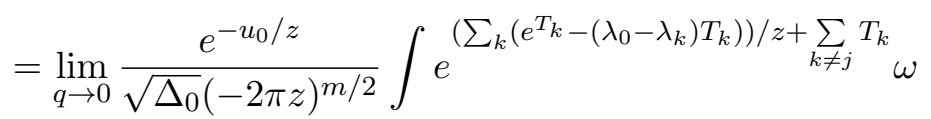

$$
\begin{aligned}
& =\lim _{q \rightarrow 0} \frac{e^{-u_{0} / z}}{\sqrt{\Delta_{0}}(-2 \pi z)^{m / 2}} \int e^{\left(\sum_{k \neq 0}\left(x_{k}-\left(\lambda_{0}-\lambda_{k}\right) T_{k}\right)+q / \prod_{k \neq 0} x_{k}\right) / z} \prod_{k \neq j} x_{j} \bigwedge_{k=1}^{m} \mathrm{~d} T_{k} .
\end{aligned}
$$

In the last step, we moved to the chart

$$
x_{0}=\frac{q}{\prod_{j \neq 0} x_{j}} .
$$

\footnotetext{
${ }^{2}$ The function $u_{i}$ of this appendix does not completely agree with the function $u_{i}$ of Section A.3. They differ by the constant $c=\sum_{j \neq i}\left(\lambda_{i}-\lambda_{j}\right)\left(-1+\ln \left(\lambda_{i}-\lambda_{j}\right)\right)$. Correspondingly, the $S_{i}$ defined via the oscillating integral also coincides with the series from localization only up to a factor $e^{c / z}$. These differences are irrelevant for the computation of the $R$-matrix.
} 


\section{Relations on $\bar{M}_{g, n}$ Via Equivariant Gromov-Witten theory of $\mathbb{P}^{1}$}

Since in this chart $\lim _{q \rightarrow 0} x_{0}=0$, we have that $R_{j 0}$ vanishes unless $j=0$. On the other hand, in the limit as $q \rightarrow 0$, the integral for $R_{00}$ splits into one-dimensional integrals

$$
\lim _{q \rightarrow 0} R_{00} \asymp \lim _{q \rightarrow 0} \frac{e^{-u_{0} / z}}{\sqrt{\Delta_{0}}(-2 \pi z)^{m / 2}} \prod_{k \neq 0} \int_{0}^{\infty} e^{\left(x-\left(\lambda_{0}-\lambda_{k}\right) \ln (x)\right) / z} \mathrm{~d} x .
$$

Let us temporarily set $z_{k}=-z /\left(\lambda_{0}-\lambda_{k}\right)$. The prefactors also split into pieces in the limit, and we calculate the factor corresponding to $k$ to be

$$
\begin{gathered}
\frac{e^{\left(1-\ln \left(\lambda_{0}-\lambda_{k}\right)\right) / z_{k}}}{\sqrt{-2 \pi z\left(\lambda_{0}-\lambda_{k}\right)}} \int_{0}^{\infty} e^{\left(x-\left(\lambda_{0}-\lambda_{k}\right) \ln (x)\right) / z} \mathrm{~d} x=\frac{e^{\left(1-\ln \left(1 / z_{k}\right)\right) / z_{k}}}{\sqrt{2 \pi / z_{k}}} \Gamma\left(1+\frac{1}{z_{k}}\right) \\
=\frac{e^{\left(1-\ln \left(1 / z_{k}\right)\right) / z_{k}}}{\sqrt{2 \pi z_{k}}} \Gamma\left(\frac{1}{z_{k}}\right) \asymp \exp \left(\sum_{l=1}^{\infty} \frac{B_{2 l}}{2 l(2 l-1)}\left(\frac{z}{\lambda_{k}-\lambda_{0}}\right)^{2 l-1}\right),
\end{gathered}
$$

using Stirling's approximation of the gamma function in the last step. So the product of the factors gives the expected limit (A.15) of $R_{00}$ for $q \rightarrow 0$.

\section{ACKnowledgements}

I thank my $\mathrm{PhD}$ advisor R. Pandharipande for all his support while I worked on this project, especially for the frequent meetings at the beginning of this project. I am also very grateful for comments from him and the anonymous referee, which led to a considerable improvement of the current text. Special thanks are due to Y. P. Lee for discussions at the conference Cohomology of the moduli space of curves organized by the Forschungsinstitut für Mathematik at ETH Zürich, which changed my way of thinking about the stable quotient relations. I am also grateful for relevant discussions with A. Marian, D. Oprea, A. Pixton and D. Zvonkine at various points of time.

\section{REFERENCES}

AC96 E. Arbarello and M. Cornalba, Combinatorial and algebro-geometric cohomology classes on the moduli spaces of curves, J. Algebraic Geom. 5 (1996), no. 4, 705-749.

BJP15 A. Buryak, F. Janda and R. Pandharipande, The hypergeometric functions of the Faber-Zagier and Pixton relations, 2015, arXiv:1502.05150.

Fab99 C. Faber, A conjectural description of the tautological ring of the moduli space of curves, in Moduli of Curves and Abelian Varieties, Aspects of Mathematics, vol. E33 (Vieweg, Braunschweig, 1999), 109-129; https://doi.org/10.1007/978-3-322-90172-9_6.

FP00 C. Faber and R. Pandharipande, Logarithmic series and Hodge integrals in the tautological ring, Michigan Math. J. 48 (2000), 215-252; https://doi.org/10.1307/mmj/1030132716.

FP05 Relative maps and tautological classes, J. Eur. Math. Soc. (JEMS) 7 (2005), no. 1, 13-49; https://doi.org/10.4171/JEMS/20.

Giv01a A. B. Givental, Gromov-Witten invariants and quantization of quadratic Hamiltonians, Mosc. Math. J. 1 (2001), no. 4, 551-568.

Giv01b , Semisimple Frobenius structures at higher genus, Int. Math. Res. Not. 2001 (2001), no. 23, 1265-1286; https://doi.org/10.1155/S1073792801000605.

GP99 T. Graber and R. Pandharipande, Localization of virtual classes, Invent. Math. 135 (1999), no. 2, 487-518; https://doi.org/10.1007/s002220050293.

GP03 Constructions of nontautological classes on moduli spaces of curves, Michigan Math. J. 51 (2003), no. 1, 93-109; https://doi.org/10.1307/mmj/1049832895. 


\section{F. JANDA}

Jan13 F. Janda, Tautological relations in moduli spaces of weighted pointed curves, 2013, arXiv:1306.6580.

Jan15_ Frobenius manifolds near the discriminant and relations in the tautological ring, 2015, arXiv:1505.03419.

KM97 M. Kontsevich and Yu. Manin, Gromov-Witten classes, quantum cohomology, and enumerative geometry, in Mirror Symmetry, II, AMS/IP Stud. Adv. Math., vol. 1 (Amer. Math. Soc., Providence, RI, 1997), 607-653; https://doi.org/10.1007/BF02101490.

LP04 Y.P. Lee and R. Pandharipande, Frobenius manifolds, Gromov-Witten theory, and Virasoro constraints, 2004, available at http://www.math.ethz.ch/ rahul/Part1.ps.

MOP11 A. Marian, D. Oprea and R. Pandharipande, The moduli space of stable quotients, Geom. Topol. 15 (2011), no. 3, 1651-1706; https://doi.org/10.2140/gt.2011.15.1651.

Mum83 D. Mumford, Towards an enumerative geometry of the moduli space of curves, in Arithmetic and Geometry, Vol. II, Progr. Math., vol. 36 (Birkhäuser Boston, Boston, MA, 1983), 271-328; https://doi.org/10.1007/978-1-4757-9286-7_12.

Pan16 R. Pandharipande, A calculus for the moduli space of curves, 2016, arXiv:1603.05151.

Pix12 A. Pixton, Conjectural relations in the tautological ring of $\bar{M}_{g, n}, 2012$, arXiv:1207.1918.

PP13 R. Pandharipande and A. Pixton, Relations in the tautological ring of the moduli space of curves, 2013, arXiv:1301.4561.

PPZ15 R. Pandharipande, A. Pixton, and D. Zvonkine, Relations on $\overline{\mathcal{M}}_{g, n}$ via 3-spin structures, J. Amer. Math. Soc.

textbf28 (2015), no. 1, 279-309; https://doi.org/10.1090/S0894-0347-2014-00808-0.

PT14 D. Petersen and O. Tommasi, The Gorenstein conjecture fails for the tautological ring of $\overline{\mathcal{M}}_{2, n}$, Invent. Math. 196 (2014), no. 1, 139-161; https://doi.org/10.1007/s00222-013-0466-z.

Tel12 C. Teleman, The structure of $2 D$ semi-simple field theories, Invent. Math. 188 (2012), no. 3, 525-588; https://doi.org/10.1007/s00222-011-0352-5.

Felix Janda felix.janda@imj-prg.fr

IMJ - PRG, UMR 7586 CNRS, UPMC, 4 Place Jussieu, 75252 Paris cedex 05, France 